UW Biostatistics Working Paper Series

3-13-1993

\title{
Breaking the Matches in a Paired T-Test for Community Interventions When the Number of Pairs is Small
}

Paula Diehr

University of Washington, pdiehr@u.washington.edu

Don C. Martin

none

Thomas D. Koepsell

University of Washington, koepsell@u.washington.edu

Allen D. Cheadle

University of Washington, cheadle@u.washington.edu

\section{Suggested Citation}

Diehr, Paula; Martin, Don C.; Koepsell, Thomas D.; and Cheadle, Allen D., "Breaking the Matches in a Paired T-Test for Community Interventions When the Number of Pairs is Small" (March 1993). UW Biostatistics Working Paper Series. Working Paper 127.

http://biostats.bepress.com/uwbiostat/paper127

This working paper is hosted by The Berkeley Electronic Press (bepress) and may not be commercially reproduced without the permission of the copyright holder.

Copyright (c) 2011 by the authors 


\title{
BREAKING THE MATCHES IN A PAIRED T-TEST \\ FOR COMMUNITY INTERVENTIONS \\ WHEN THE NUMBER OF PAIRS IS SMALL
}

\author{
Paula Diehr, Ph.D. ${ }^{1,2}$ \\ Donald C Martin, Ph.D. ${ }^{1}$ \\ Thomas Koepse11, M.D., M.P.H. 2,3 \\ Allen Cheadle, Ph.D. ${ }^{2}$
}

From the Departments of (1) Biostatistics, (2) Health Services, and (3) Epidemiology of the University of Washington.

Supported by a grant from the Henry J. Kaiser Family Foundation and Grant Number CA 34847 from the National Cancer Institute.

Submitted to Statistics in Medicine 
http://biostats.bepress.com/uwbiostat/paper127 


\section{Abstract}

There is considerable interest in community interventions for health promotion, where the community is the experimental unit. Because such interventions are expensive, the number of experimental units (communities) is usually very small. Because of the small number of communities involved, investigators often match treatment and control communities on demographic variables before randomization to minimize the possibility of a bad split. Unfortunately, matching has been shown to decrease the power of the design when the number of pairs is very small, unless the matching variable is very highly correlated with the outcome variable (in this case, with change in the health behavior). We used computer simulation to examine the performance of an approach in which communities were matched but an unmatched analysis was performed. If the appropriate matching variables are unknown, and there are fewer than ten pairs, an unmatched design and analysis has the most power. However, if a matched design is strongly preferred then for $\mathrm{N}<10$, power is increased by performing an unmatched analysis of the matched data. A variant of this procedure, in which an unmatched analysis is performed only if the matching "didn't work", is also discussed. 


\section{Introduction}

A recent trend in health promotion is the development of interventions that target an entire community, such as community-based health promotion. Controlled evaluations of such interventions typically compare a group of intervention communities with a group of control communities in terms of reducing the prevalence of an unhealthy behavior, such as smoking. The number of communities has usually been small, for budgetary reasons: 6 for the Minnesota Heart Health Program (3 treatment and 3 control) [1], just 2 for North Karelia Project [2] and the Pawtucket Heart Health Program [3], and 3 and 5 for two studies at Stanford $[4,5,6,7,8]$. Community intervention studies are also described by shea and Basch [9] .

An important design issue in such evaluations is whether to use simple random allocation to form the comparison groups, or to form matched pairs of communities and then randomly assign the intervention to one community from each pair. Because the number of communities is small, a "bad" randomization, such as having all of the biggest communities, in the treatment group, is likely to occur. This possibility often causes evaluators to choose a matched design, even in the absence of knowledge about appropriate matching variables.

The dependent variable of interest is usually change in some health behavior, such as change in the percent of residents who smoke cigarettes. It is common in the literature to find information on correlates of smoking behavior at the individual level; there is also some individual-level information about correlates of change in smoking. 
"There is, however, relatively little published information about correlations between community characteristics and smoking prevalence, and almost no information about the correlation between community characteristics and the change in smoking prevalence. Even if such estimates were available they would usually be of poor quality, since the correlations would usually be based on a very small number of communities.

Unfortunately, the situation where the number of pairs is small and the appropriate matching variables are unknown is exactly where a matched test can be shown to have lower power than an unmatched test [10]. This occurs because of the low number of degrees of freedom for the matched design. Since power is allready low due to the small number of communities, the issue of whether to use a matched versus an unmatched design is clearly important.

This preference for matching, in a situation. where power is reduced by matching, suggests a hybrid design in which one would match, but then analyze the data as though the matching had not occurred. As will be illustrated below, breaking matches is usually conservative, in that it makes it harder to reject the null hypothesis $[11,12]$. However, when the number of experimental units is very small, such a procedure may be worth considering. The goal of this paper is to examine the situation in which communities are matched, prior to randomization, on a variable, $x$, which has correlation $\rho_{x y}$ with the outcome variable, $Y$ (e.g., change in smoking rates). We then compare the power for the correct matched analysis to two variants in which matched data receive an unmatched analysis all or some of the time. The power of a 
completely unmatched design is also compared to that of the three matched designs.

This problem is not analytically tractable because the distribution of the unmatched $t$ statistic calculated from matched data does not reduce to the usual ratio of a normal variate over the square root of an independent chi-square variate. (Normality holds, but the chi-square and independence assumptions fail). For this reason we conducted a computer simulation to estimate the power of different approaches for various numbers of pairs, values of $\rho_{x y}$, and effect sizes.

\section{Background}

Review of Matched and Unmatched t-tests

Table 1 shows hypothetical data for 2 groups, with 4 observations per group. We assume that $\sigma_{1}=\sigma_{2}$. If the design was unmatched, the t-statistic would be

$$
t_{\text {unmatched }}=\frac{\overline{y_{1}}-\overline{y_{2}}}{\sqrt{\frac{S_{1}{ }^{2}}{N}+\frac{S_{2}{ }^{2}}{N}}}=\frac{\bar{d}}{S_{u}}=2.56
$$

and if matched it would be

$$
t_{\text {matched }}=\frac{\bar{d}}{\frac{S_{d}}{\sqrt{N}}}=\frac{\bar{d}}{S_{m}}=2.51
$$

where $\bar{y}$ and $\bar{d}$ are sample means, and $s$ is the sample standard deviation. In the unmatched test we would reject if $t_{u}>2.4$ ( 6 degrees of 
- freedom) and in the matched test we would reject if $t_{m}>3.18$ ( 3 df). For the data of Table 1, the unmatched test rejects the null hypothesis but the matched test does not.

\section{[Table 1 about here]}

Two different correlation coefficients are relevant: $r_{\mathrm{XY}}$ and $r_{\mathrm{YY}}$. The first is the correlation between $X$, the matching variable, and $Y$, the dependent variable (for example, the correlation between community size and. change in smoking prevalence). The second, $r_{y y}$ is the correlation on the outcome variable between the members of a pair ( $Y_{1}$ and $\mathrm{Y}_{2}$ ). If the match is perfect (the value of $\mathrm{X}$ is identical for both members of the pair), then $r_{x y}^{2}=r_{y y}$.

To give some insight into the performance of the matched and unmatched t-tests when $N$ is small, consider the rejection region for the tests:

- Unmatched: $\quad$ reject if $\bar{d}>2.45 \mathrm{~S}_{\mathrm{u}}$

and

Matched: $\quad$ reject if $\overline{\mathrm{d}}>3.18 \mathrm{~S}_{\mathrm{m}}$

If $2.45 \mathrm{~S}_{\mathrm{u}}=3.18 \mathrm{~S}_{\mathrm{m}}$, then the tests are equivalent; otherwise, one requires a bigger values of $\bar{d}$ than the other. An alternative way of expressing $S_{m}$ is

$$
S_{m}^{2}=\frac{S_{1}{ }^{2}}{N}+\frac{S_{2}{ }^{2}}{N}-\frac{2 r_{y \gamma} S_{1} S_{2}}{N}
$$


If $S_{1}$ in the sample is approximately equal to $S_{2}$, then $S^{2}$ is approximately equal to $S_{u}^{2}\left(1-r_{y y}\right)$. In this case, $2.45 S_{u}$ is equal to $3.18 \mathrm{~S}_{\mathrm{m}}$ when

$$
(2.45)^{2} \quad S_{u}^{2}=(3.18)^{2} \quad S_{u}^{2}\left(1-r_{Y Y}\right),
$$

or

$$
r_{\mathrm{YY}}=1-(2.45 / 3.18)^{2}=.406 .
$$

That is, the matched test is more likely to reject the null hypothesis than the unmatched test if $r_{y y}$ is larger than .406 . Otherwise the unmatched test is more likely to reject. More generally, if $s_{1}$ is about equal to $s_{2}$, the matched and unmatched tests are about the same if

$$
r_{Y Y}=1-(\mathrm{CV}(\mathrm{u}) / \mathrm{CV}(\mathrm{m}))^{2}
$$

where $\mathrm{CV}(\mathrm{u})$ and $\mathrm{CV}(\mathrm{m})$ represent the unmatched and matched critical values, respectively. Table 2 shows some critical values, and the value of the correlation at which we would be indifferent between the matched and the unmatched tests. For example, for 2 pairs, the two critical values are 12.7 and 4.3 , and the tests would be the same if $r_{x y}=.94$, or equivalently, if $r_{y y}=.88$. Clearly, $r_{x y}$ would have to be very high before we would choose a matched test for smaller samples only to improve power.

[Table 2 about here]

Martin [10] computed the break-even correlation based on population parameters, $\rho_{x y}$ or $\rho_{y y}$, which similarly showed that matched tests were less powerful than unmatched tests for very small numbers of pairs unless $\rho_{x y}$ was extremely high.

\section{Design Strategies}


and In community interventions there are thus good reasons for and against matching, both intensified by the small number of communities available. Is it possible to have a matched design without losing power? Table 3 shows four possible strategies, depending on whether the design is matched or unmatched, and whether the analysis is matched or unmatched. The first strategy, labelled "UU", has an unmatched design and an unmatched analysis, and is the usual unmatched t-test. The second, MM, is the usual matched t-test. The third design, Mo, represents a matched design with an unmatched analysis. The fourth, MUT (matched/unmatched with testing), is a variant in which the matched data are first tested to see whether $r_{y y}$ is significantly greater than zero (one-tailed, $\alpha=.05$ ). If $r_{y y}$ is significant, a matched analysis is performed; otherwise an unmatched analysis is used.

[Table 3 about here]

The goal of this paper is to evaluate the MU and MUT strategies. We are interested first in whether these procedures are legitimate (yield the correct $\alpha$ level when the null hypothesis is true), and second in whether they improve power. We performed a computer simulation to evaluate the $\alpha$ level and the power of these two methods.

\section{METHODS}

The statistical model assumed for the simulation is as follows: let $X_{1}, e_{1 i}$, and $e_{2 i}$ be independent random variables chosen from a normal distribution with mean 0 and variance 1.

Let $\quad Y_{1 i}=\rho_{x y} X_{i}+\sqrt{1-\rho^{2} x y} e_{1 i}$ 


$$
Y_{2 i}=\Delta+\rho_{x y} X_{i}+\sqrt{1-\rho_{x y}^{2}} e_{2 i}
$$

where $Y_{1 i}$ is the simulated outcome variable for the control member of pair $i, Y_{2 i}$ is the outcome for the treatment member, and $\Delta$ is the treatment effect size. Then

$$
\begin{gathered}
Y_{1} \sim N\left(0, \rho_{x y}^{2}+1-\rho_{x y}^{2}\right)=N(0,1) \\
Y_{2} \sim N(\Delta, 1)
\end{gathered}
$$

and

$$
\operatorname{Corr}(X, Y)=\rho_{X Y}
$$

and

$$
\operatorname{Corr}\left(Y_{1}, Y_{2}\right)=\rho_{Y Y}=\rho_{X Y}^{2} \text {. }
$$

(The last relationship can be understood by noting that the proportion of variance in $X$ explained by $Y_{1}$ is $\rho^{2}{ }_{x y}$, and the proportion of the variation in $\mathrm{Y}_{2}$ explained by $\mathrm{X}$ is also $\rho_{\mathrm{xy}}^{2}$; the proportion of variability of $Y_{1}$ explained by $Y_{2}$ is thus $\left(\rho_{X Y}^{2}\right)^{2}$, or $\left(\rho_{Y Y}\right)^{2}=\left(\rho_{x y}^{2}\right)^{2}$. Here, the match on $\mathrm{X}$ is perfect. If not, then $\rho_{\mathrm{yy}}<\rho_{\mathrm{xy}}^{2}$ ).

The simulation is based on standardized variables. For nonstandardized variables, the effect size is

$$
\Delta=\left(\mu_{y_{1}}-\mu_{y_{2}}\right) / \sigma_{\gamma} .
$$

To estimate the power of the different t-tests, we first created a sample of $\mathrm{N}$ pairs, as above. For this sample we calculated $t_{\mathrm{mm}}, t_{\mathrm{mu}}$, and $t_{\text {mu }}$ and determined whether or not the null hypothesis was rejected by each method. We repeated this process 100,000 times, and counted the 
-proportion of times the null hypothesis was rejected; this is an estimate of the power of the test. The simulations were done for parameter values $N=2$ to $20, \rho_{x y}=0$ to .9 , and $\Delta=$ effect size $=0$ to 3. The power of UU is the same as the power of $\mathrm{MU}$ when $\rho_{\mathrm{xy}}=0$. With 100,000 iterations for each situation, the 95\% confidence interval for power has width \pm .001 when the true power is .05 , and \pm .003 when the true power is .50 .

In preliminary runs, we found that the power of MUT when $\Delta=0$ (that is, the estimated $\alpha$ level) was greater than .05 . This is not surprising, since the method of analysis was chosen after peeking at the data. Without some correction, MUT is an inappropriate test, since it will reject too often when the null hypothesis is true. In order to study MUT further, we estimated the critical values necessary to give it $\alpha=, 05$. This was done by generating 100,000 values of the MUT $t-$ statistic, and using the 97.5 th percentile of those data as an estimate of the critical value for the MUT distribution. The results presented below all use the estimated critical values.

\section{RESOLTS}

The estimated critical values for the MUT distribution are presented. We then consider the power under two situations: the null hypothesis and the alternative hypothesis. Results for $\rho_{x y}=0$, and $\rho_{x y}$ $>0$ are discussed separately.

MUT Critical Values

The estimated critical values for the MUT procedure are in Table 4. The MUT procedure was to calculate the matched t-statistic if $r_{Y y}$ is 
significantly greater than zero, the unmatched t-statistic otherwise, and to reject the null hypothesis if $t_{\text {mut }}$ is greater than the number in Table 4 .

\section{[Table 4 about here]}

\section{Null Hypothesis True}

Table 5 shows the estimated power when $\Delta=0$ and $\rho_{x y}=0$; that is, there is no treatment effect and the matching variable is completely uncorrelated with $\mathrm{Y}$. In this null situation, power is the same as the $\alpha$ level, and the power of MM, UU, and MU are all .05. The power of MUT' (the MUT analysis using the usual critical values) is always greater than .05 , but the power of MUT, which uses the estimated critical values of Table 4 is appropriately about .05. MUT' is not considered further. [Table 5 about here]

The $\alpha$ levels of MM and UU are .05, by design. It may seem surprising that the $\alpha$ level of MU is also .05, since the analysis does not correspond to the design. However, when $\rho_{\mathrm{xy}}=0$, both MU and MM are appropriate because the matching had no effect. Equation $\{1\}$, above, shows that when $\rho_{x y}=0, Y_{1 i}$ and $Y_{2 i}$ are completely independent, and so can be considered to be unmatched. To understand further how both MU and MM can be appropriate, consider the following formulation of the two $t$ statistics.

$$
t_{\text {unmatched }}=\frac{\bar{d}}{\sqrt{\frac{s_{1}{ }^{2}}{N}+\frac{s_{2}{ }^{2}}{N}}}, d f=2(N-1)
$$

and 


$$
t_{\text {matched }}=\frac{\bar{d}}{\sqrt{\frac{s_{1}^{2}}{N}+\frac{s_{2}{ }^{2}}{N}-\frac{2 r_{y \gamma} s_{1} s_{2}}{N}}}, d f=N-1
$$

The two statistics differ only in the last term under the square root for $t_{m}$. Although $\rho_{Y y}=0$, and $r_{Y Y}$ is 0 on average, $r_{Y y}$ is often very large when the number of pairs is small; for $\mathrm{N}=2$ pairs, for example, $r_{Y Y}$ is always plus or minus 1. Both of the t-test denominators are the same, "on average", but $S_{m}$ is more variable than $S_{u}$, because of the final term which has mean zero and adds no information. Both $S_{m}$ and $S_{u}$ (squared) can be shown to have a chi-squared distribution. Since $\mathrm{S}_{\mathrm{m}}$ is more variable than $S_{u}$, the chi-square statistic based on $S_{m}$ must have fewer degrees of freedom, and the corresponding t-test must also have fewer degrees of freedom.

Table 6 provides estimated $\alpha$ level (power) estimates when $\rho_{x y}>0$ but $\Delta=0$; that is, when the matching variable is correlated with the outcome variable, but there is no treatment effect. The $\alpha$ level of MM and UU remain at .05, as would be expected. The $\alpha$ level of MU decreases as $\rho_{x y}$ increases, and also for larger $N$. This is why breaking matches is usually considered to be disadvantageous $[11,12]$. The $\alpha$ level of $\mathrm{ML}$ is considerably below .05 in some cases. The $\alpha$ level for MUT, however, is always close to .05 . This is because MUT performs the matched analysis when $r_{y y}$ is significantly different from zero. MUT is identical to MU for 2 pairs, since $r_{x y}$ is always plus or minus 1 , and can not be significantly different from zero. All of these methods are legitimate, since the $\alpha$ level is .05 or less in every case.

[Table 6 about here] 
Nu11 Hypothesis False

We next consider power when the treatment effect is not zero. Table 7 shows power estimates when the alternative hypothesis is true, for selected values of $N, \rho_{x y}$ and $\Delta$. Complete simulation results, for all values of parameters, are available elsewhere [13]. The estimated power of MM, UU, MU, and MUT are shown for each situation. As would be expected, power increases as $\Delta$ or $\mathrm{N}$ get larger for all strategies, and as $\rho_{x y}$ increases for $M M$. Note that for $N=2$ pairs, power is always very low. Even with an effect size of 3, power is less than .4. The power for $N=3$ pairs is also quite low. For other values of $N$, if $\Delta$ is high, the power can be near to 1 .

There are situations in which each method excels. For example, for 4 pairs, with $\rho_{x y}=0$ and $\Delta=1.0, \mathrm{MU}$ and UU have higher power than $\mathrm{MM}$ and MUT. For $\rho_{x y}=.80$, and $\Delta=.5$, however, $M$ M and MUT are superior to MU and UU. For $\rho_{x y}=.80$, and $\Delta=1.0, \quad M M$ is superior to the other methods.

[Table 7 about here]

\section{SUMMARY AND DISCUSSION}

A reasonable summary recommendation is that, for 2 pairs, one should resist doing the experiment since power is very low. If it must be done, use UU or, if matching is avoidable, use MU. A matched analysis is always worst (but all methods have very low power). For $3-9$ pairs, either UU or MU should be chosen. For 10 to 20 pairs, MM should be chosen, since it is usually as good as the other methods and will be best in the rare situation in which a very good correlate is available 
for matching. These recommendations do not, of course, cover every situation. Readers who need more detail should refer to Table 7 , or to the more complete tables [13].

The simulation results would be more helpful if reasonable estimates of $\rho_{x y}$ and $\Delta$ were available. Unfortunately, there is little information available about which community-level variables are correlated with change over time in health behaviors for that community. We computed the correlation of six community characteristics (percent of residents who were male, white, black, asian, high school graduate, or low income), with changes over two years in two health behaviors (smoking prevalence and mean percent calories from fat) for 13 communities in the Henry $J$ Kaiser Family Foundation Community Health Promotion Grants Program (CHPGP) [14]. The largest correlation, between percent black and change in smoking prevalence, was only .59, with a 95\% confidence interval from .058 to .862 . This provides scant evidence of large community correlations, especially if one adjusts for multiple comparisons. Since most community programs have small numbers of communities, other estimates of correlations are likely to be equally unsatisfying.

It is also difficult to estimate how large $\Delta$, the effect size, will be. If $Y$ is the change in a health behavior (e.g., the change in smoking prevalence), then $\sigma_{y}$ from equation [2] has two components: variation among communities in the true rate, and a sampling error which is small if very large samples are taken in each community. In the CHPGP data set we found that the standard deviation of change in smoking prevalence was about 3.5 percentage points for 13 communities [14]. An 
effect size of $\Delta=1$ would thus require a treatment to decrease smoking prevalence by about 3.5 percentage points, from a baseline average prevalence of about $24 \%$, while the control group did not change. (Or, that the treatment group changed 3.5 percentage points more than the control group). This would require an unusually vigorous community intervention. The values for $\Delta=2$ or 3 (requiring 7 and 10.5 percentage point decreases respectively) seem fanciful.

In most situations, it is unlikely that we are lucky enough to match on a good variable, and it is further unlikely that the effect size is bigger than 1. Restricting attention to results for $\rho_{x y}<.6$ and $\Delta \leq 1$, Table 7 shows that it doesn't matter very much what rule is used. These results generalize to matching on more than one covariate. In this case multiple $R$ plays the role of $r_{x y}$.

There are some limitations to this research. It was restricted to the situation where $\sigma_{1}=\sigma_{2}$, and to normally distributed data. These assumptions are common for power studies. We briefly considered a log normal distribution for $x$. Preliminary results were similar to those already shown. Perhaps the most notable finding was that the $\alpha$ level was .05 for $M M$ and $U U$, in spite of the skewed distribution and the small number of pairs. This robustness of the t-statistic to departures from normality is not always fully appreciated. We have not explored this aspect fully since there is little guidance on how best to model changes over time in community behaviors.

The performance of the MUT approach may be better than we have shown. We defined a "helpful" match as one in which $r_{y y}$ was significantly greater than zero. Other definitions, such as $r_{y y}>.5$ or using a lower 
. $\alpha$ would have had different power results. Ideally, MUT would look like UU for low values of $\rho_{y y}$ and like $M$ M for high values of $\rho_{x y}$. The estimated critical values for MUT may have been a little high, since power in Table 5 is always slightly below .05; this would understate the power of MUT somewhat. MUT is less attractive than other methods, however, since it can not use the usual tabled critical values.

Results were presented in terms of $\rho_{x y}$ because it is easier to obtain estimates of $\rho_{x y}$ than of $\rho_{Y y}$. However, the tabled results are correct only if we are able to achieve a perfect match on $\mathrm{X}$, which is unlikely in real practice. If matches are imperfect, the correspondence between $\rho_{x y}^{2}$ and $\rho_{y y}$ is not exact, and $\rho_{x y}$ will have to be even larger than the tabled value. The tables remain correct if the label $\rho_{x y}$ is replaced by $\left(\rho_{y y}\right)^{.5}$.

These findings are valuable because important studies must sometimes be done using very small numbers of experimental units. It is distressing to find conflicting guidelines for how those few units should be allocated. It is encouraging to find that a "common sense" solution to a problem, such as the MU approach, is also reasonable from a statistical perspective. 


\section{Bibliography}

1. Blackburn H, Lueker RV, Kline FG, Bracht $N$, Carlow R, Jacobs D, Stauffer L, Taylor HL. The Minnesota Heart Health Program: A research and demonstration project in cardiovascular disease prevention. In Matarazzo JD, Weiss SM, Miller NE and Weiss SW (eds.). A Handbook of Health Enhancement and disease Prevention. Wiley, New York, 1984, pp. 1171-1178.

2. McAlister A, Puska P, Salonen J, Tuomilehto J, Koskela K. Theory and action for health promotion: illustrations from the North Karelia project. American Journal of Public Health 72:43-50. 1982 .

3. Lefebvre R, Lasater TM, Carlton RA, Peterson G. Theory and delivery of health programming in the community: the Pawtucket Heart Health program. Preventive Medicine 16:80-95 1987.

4. Farquhar JW, Fortmann SP, MacCoby N, Haskell WL, Williams PT, Flora $J A$, Taylor CB, Brown BW Jr., Solomon DS, Hulley SB. The Stanford five-city project: Design and methods. American Journal of Epidemiology, 122(2):323-334. 1985.

5. Fortman SP, Williams PT, Hulley SB, Haskell Wl, Farquhar JW. Effect of health education on dietary behavior: the Stanford three community study. American Journal of Clinical Nutrition 34:20302038. 1981.

6. Fortmann SP, Haskell WL, Williams PT, Varady. AN, Hulley SB, Farquhar JW. Community surveillance of cardiovascular diseases in the Stanford five-city project. Methods and initial experience. American Journal of Epidemiology 123(4):656-669. 1986.

7. Sallis JF, Haskell WL, Wood PD, Fortmann SP, Rogers T, Blair SN, Paffenbarger.RS. Physical activity assessment methodology in the five-city project. American Journal of Epidemiology 121(1):91-106. 1985 .

8. Williams PT, Fortmann SP, Farquhar JW, Varady A, Mellen SJ. A comparison of statistical methods for evaluating risk factor changes in community-based studies: an example from the Stanford Three Community Study. Chronic Disease 34:565-571. 1981.

9. Shea S, Basch CE. A review of five major community-based cardiovascular disease prevention programs. Part I: Rationale, design, and theoretical framework. American Journal of Health Promotion 4(3):203-213. 1990. 
10. Martin DC, Diehr P, Perrin E, Koepsell T. The effect of matching on the power of randomized community intervention studies. Statistics in Medicine, 1993;12:329-338.

11. Snedecor GW, Cochran WG. Statistical Methods, 6th edition, Iowa State University Press, Ames, Iowa, 1979. page 108.

12. Lynn HS, MCCulloch CE. When does it pay to break the matches for analysis of a matched-pairs design? Biometrics 48:397-409. 1992.

13. See Appendix 1.

14. Wagner E, Koepsell T, Anderman C, Cheadle A, Curry S, Psaty B, Wickizer T, Beery W, Diehr P, Ehreth J, Kehrer B, Pearson D, Perrin E, VonKorff M: The evaluation of the Kaiser Family Foundation's health promotion grants program: Overall design. Journal of Clinical Epidemiology 44:685-699, 1991. 
Table 1

Review of a t-test

$\begin{array}{rll}Y_{11} & Y_{21} & d_{1} \\ 6 & 3 & 3 \\ 11 & 4 & 7 \\ 10 & 5 & 5 \\ 6 & 6 & 0 \\ 8.25 & 4.5 & 3.75 \\ 2.63 & 1.29 & 2.99 \\ 4 & 4 & 4\end{array}$

n

mean

sd 


\section{Table 2}

\section{Critical Values and Breakpoint Sample Correlations for Matched an Unmatched t-tests*}

N

MATCHED

UNMATCHED

$r_{Y Y}$

$r_{x y}$

df $\mathrm{cv}(\mathrm{m})$ df $\mathrm{cv}(\mathrm{u})$

2

12.7

2

4.3

.88

.94

3

2

4.3

4

2.8

.58

.76

4

$3 \quad 3.2$

$6 \quad 2.4$

.41

.66

5

10

42.8

8

2.3

.32

.57

92.3

18

2.1

.17

.41

20

192.1

38

2.02

.07

.27

$\infty \quad 1.96$

$\infty \quad 1.96$

0

0

The breakpoint correlation is the value of $r$ for which the matched and unmatched $t$-tests are the same (assuming $s_{1}$ approximately equal to $s_{2}$ for this particular sample. 


\section{Table 3}

\section{Four Strategies for Design and Analysis}

DESIGN

$\mathrm{U}$

M

$\mathrm{M}$

M

\section{ANALYSIS COMMENTS}

U

M

$\mathrm{U}$

UT
Standard Unmatched t-test

Standard Matched t-test

Matched data, perform unmatched t-test

Matched data, test whether $r_{Y y}$ is significantly greater than zero, perform unmatched $t$-test unless $r_{Y y}$ is significant. 
Table 4

\section{Estimated Critical values for MUT*}

\section{$N$ of Critical \\ Pairs Value}

2

3

4

5

6

7

8

9

10

11

12

13

14

15

16

17

18

19

$-20$
4.3027

3.6460

2. 8180

2.5463

2.3813

2. 3429

2. 2473

2. 2045

2.1991

2. 1552

2. 1564

2. 1149

2.0989

2.1018

2.1172

2.0867

2. 0732

2.0508

2.0586

* Each critical is the 97.5 th percentile of 100,000
generated at random from the MUT' distribution.

* Each critical is the 97.5 th percentile of 100
generated at random from the MUT' distribution.

t-statistics 


\section{Table 5}

$\alpha$ Level or Power when the effect is zero and $\rho_{x y}=0$

$\begin{array}{rcccccc}\begin{array}{l}\text { N of } \\ \text { Pairs }\end{array} & \rho_{\mathbf{x y}} & \text { UU } & \text { MM } & \text { MU } & \text { MUT } & \text { MUT' }^{\prime} \\ 2.00 & .00 & .051 & .051 & .051 & .051 & .051 \\ 3.00 & .00 & .050 & .049 & .050 & .045 & .070 \\ 4.00 & .00 & .049 & .050 & .049 & .046 & .065 \\ 5.00 & .00 & .052 & .051 & .052 & .047 & .065 \\ 6.00 & .00 & .050 & .050 & .050 & .048 & .061 \\ 8.00 & .00 & .049 & .050 & .049 & .048 & .058 \\ 10.00 & .00 & .049 & .049 & .049 & .046 & .056 \\ 15.00 & .00 & .050 & .050 & .050 & .048 & .055 \\ 20.00 & .00 & .051 & .050 & .051 & .050 & .055\end{array}$

MUT' is the power of the test with unadjusted critical values. MUT uses the critical values from Table 4. 
Table 6

$\alpha$ Level or Power when Effect Size is zero and $\rho_{x y}>0$

\begin{tabular}{|c|c|c|c|c|c|c|}
\hline $\begin{array}{l}\text { N of } \\
\text { Pairs }\end{array}$ & & $\rho_{x y}$ & UU & $\mathrm{MM}$ & MO & MUT \\
\hline $\begin{array}{r}2.00 \\
3.00 \\
4.00 \\
5.00 \\
6.00 \\
8.00 \\
10.00 \\
15.00 \\
20.00\end{array}$ & & $\begin{array}{l}.20 \\
.20 \\
.20 \\
.20 \\
.20 \\
.20 \\
.20 \\
.20 \\
.20\end{array}$ & $\begin{array}{l}.051 \\
.050 \\
.049 \\
.052 \\
.050 \\
.049 \\
.049 \\
.050 \\
.051\end{array}$ & $\begin{array}{l}.050 \\
.050 \\
.050 \\
.050 \\
.050 \\
.050 \\
.050 \\
.049 \\
.050\end{array}$ & $\begin{array}{l}.047 \\
.047 \\
.047 \\
.046 \\
.047 \\
.047 \\
.047 \\
.045 \\
.047\end{array}$ & $\begin{array}{l}.047 \\
.044 \\
.045 \\
.045 \\
.047 \\
.047 \\
.045 \\
.045 \\
.047\end{array}$ \\
\hline $\begin{array}{r}2.00 \\
3.00 \\
4.00 \\
5.00 \\
6.00 \\
8.00 \\
10.00 \\
15.00 \\
20.00\end{array}$ & & $\begin{array}{l}.40 \\
.40 \\
.40 \\
.40 \\
.40 \\
.40 \\
.40 \\
.40 \\
.40\end{array}$ & $\begin{array}{l}.051 \\
.050 \\
.049 \\
.052 \\
.050 \\
.049 \\
.049 \\
.050 \\
.051\end{array}$ & $\begin{array}{l}.049 \\
.051 \\
.050 \\
.050 \\
.050 \\
.050 \\
.049 \\
.051 \\
.050\end{array}$ & $\begin{array}{l}.043 \\
.040 \\
.037 \\
.036 \\
.035 \\
.036 \\
.034 \\
.034 \\
.034\end{array}$ & $\begin{array}{l}.043 \\
.044 \\
.042 \\
.042 \\
.043 \\
.042 \\
.039 \\
.040 \\
.041\end{array}$ \\
\hline $\begin{array}{r}2.00 \\
3.00 \\
4.00 \\
5.00 \\
6.00 \\
8.00 \\
10.00 \\
15.00 \\
20.00\end{array}$ & & $\begin{array}{l}.60 \\
.60 \\
.60 \\
.60 \\
.60 \\
.60 \\
.60 \\
.60 \\
.60\end{array}$ & $\begin{array}{l}.051 \\
.050 \\
.049 \\
.052 \\
.050 \\
.049 \\
.049 \\
.050 \\
.051\end{array}$ & $\begin{array}{l}.050 \\
.050 \\
.050 \\
.051 \\
.049 \\
.050 \\
.050 \\
.050 \\
.049\end{array}$ & $\begin{array}{l}.035 \\
.029 \\
.024 \\
.022 \\
.020 \\
.019 \\
.018 \\
.017 \\
.016\end{array}$ & $\begin{array}{l}.035 \\
.046 \\
.043 \\
.041 \\
.041 \\
.039 \\
.038 \\
.040 \\
.040\end{array}$ \\
\hline $\begin{array}{r}2.00 \\
3.00 \\
4.00 \\
5.00 \\
6.00 \\
8.00 \\
10.00 \\
15.00 \\
20.00\end{array}$ & . & $\begin{array}{l}.80 \\
.80 \\
.80 \\
.80 \\
.80 \\
.80 \\
.80 \\
.80 \\
.80\end{array}$ & $\begin{array}{l}.051 \\
.050 \\
.049 \\
.052 \\
.050 \\
.049 \\
.049 \\
.050 \\
.051\end{array}$ & $\begin{array}{l}.051 \\
.049 \\
.049 \\
.049 \\
.050 \\
.050 \\
.049 \\
.049 \\
.050\end{array}$ & $\begin{array}{l}.024 \\
.014 \\
.010 \\
.007 \\
.007 \\
.005 \\
.003 \\
.002 \\
.002\end{array}$ & $\begin{array}{l}.024 \\
.049 \\
.047 \\
.047 \\
.049 \\
.049 \\
.048 \\
.050 \\
.052\end{array}$ \\
\hline
\end{tabular}




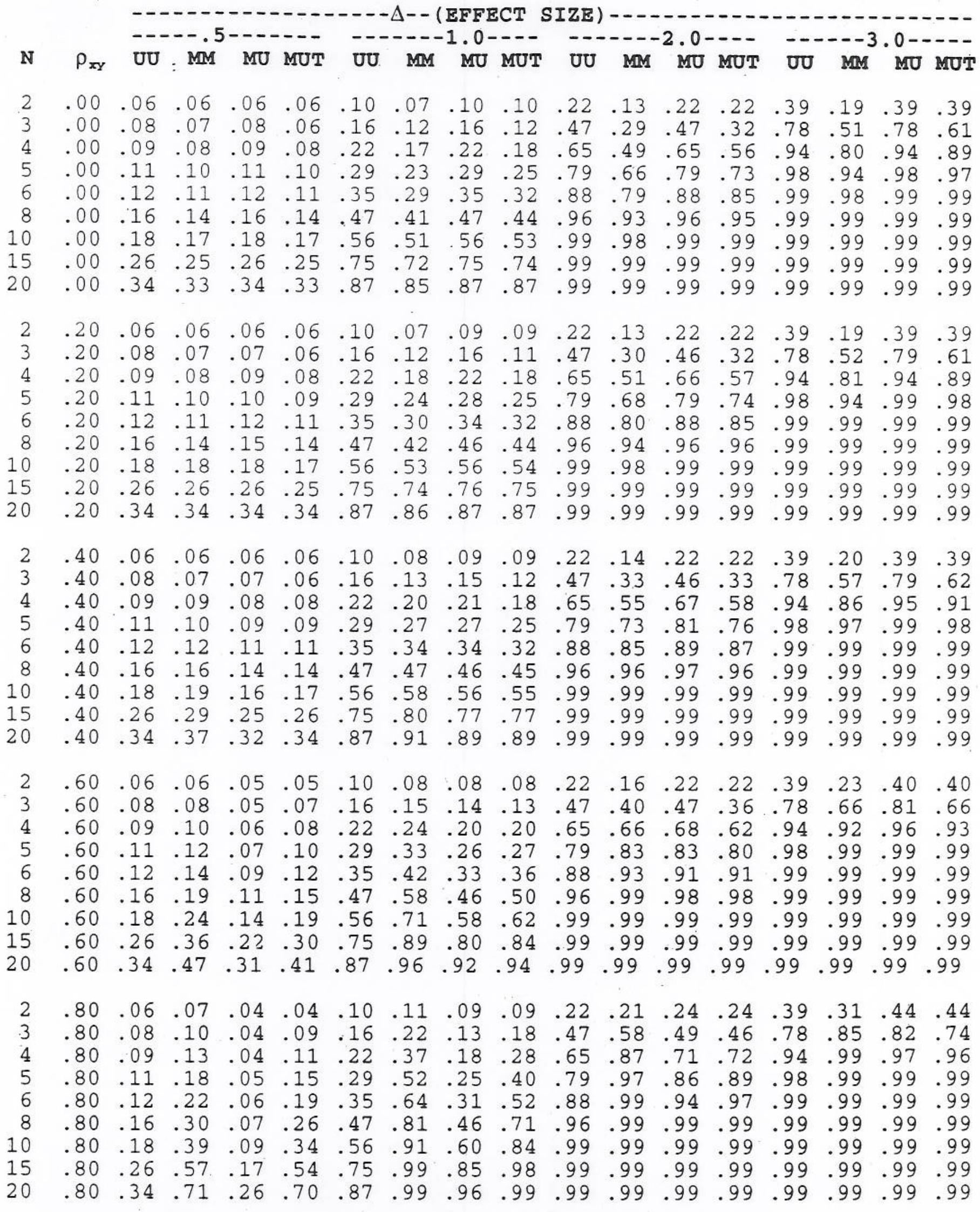


Appendix 1

Complete Simulation Results

$5-3-93$

cutoff for MUT based on 100,000 calculations 
POWER FOR 2 PAIRS

\begin{tabular}{|c|c|c|c|c|c|c|c|c|c|c|c|}
\hline$\rho_{x y} \quad \mathrm{ME}$ & OD & .0 & .50 & 1.00 & 1.50 & 2.00 & 2.50 & 3.00 & 3.50 & 4.00 & 4.50 \\
\hline $\begin{array}{l}.00 \\
.00 \\
.00 \\
.00\end{array}$ & $\begin{array}{l}\text { MM } \\
\text { MU } \\
\text { MUT } \\
\text { UU }\end{array}$ & $\begin{array}{l}.051 \\
.051 \\
.051 \\
.051\end{array}$ & $\begin{array}{l}.056 \\
.062 \\
.062 \\
.062\end{array}$ & $\begin{array}{l}.074 \\
.096 \\
.096 \\
.096\end{array}$ & $\begin{array}{l}.097 \\
.150 \\
.150 \\
.150\end{array}$ & $\begin{array}{l}.126 \\
.220 \\
.220 \\
.220\end{array}$ & $\begin{array}{l}.157 \\
.301 \\
.301 \\
.301\end{array}$ & $\begin{array}{l}.187 \\
.388 \\
.388 \\
.388\end{array}$ & $\begin{array}{l}.217 \\
.478 \\
.478 \\
.478\end{array}$ & $\begin{array}{l}.247 \\
.566 \\
.566 \\
.566\end{array}$ & $\begin{array}{l}.2 \\
.6 \\
.6\end{array}$ \\
\hline $\begin{array}{l}.10 \\
.10 \\
.10\end{array}$ & $\begin{array}{l}\text { MM } \\
\text { MU } \\
\text { MUT }\end{array}$ & $\begin{array}{l}.050 \\
.050 \\
.050\end{array}$ & $\begin{array}{l}.056 \\
.062 \\
.062\end{array}$ & $\begin{array}{l}.073 \\
.095 \\
.095\end{array}$ & $\begin{array}{l}.097 \\
.149 \\
.149\end{array}$ & & & & & $\begin{array}{l}.247 \\
.564 \\
.564\end{array}$ & .6 \\
\hline $\begin{array}{l}.20 \\
.20 \\
.20\end{array}$ & $\begin{array}{l}\text { MM } \\
\text { MU } \\
\text { MUT }\end{array}$ & .04 & $\begin{array}{l}.056 \\
.058 \\
.058\end{array}$ & & $\begin{array}{l}.099 \\
.146 \\
.146\end{array}$ & & .158 & & & .25 & \\
\hline $\begin{array}{l}.30 \\
.30 \\
.30\end{array}$ & $\begin{array}{l}\text { MM } \\
\text { MU } \\
\text { MUT }\end{array}$ & $\begin{array}{l}.050 \\
.047 \\
.047\end{array}$ & $\begin{array}{l}.056 \\
.059 \\
.059\end{array}$ & & $\begin{array}{l}.101 \\
.146 \\
.146\end{array}$ & & & & & 25 & $\begin{array}{l}.2 \\
.6 \\
.6\end{array}$ \\
\hline $\begin{array}{l}.40 \\
.40 \\
.40\end{array}$ & $\begin{array}{l}\text { MM } \\
\text { MU } \\
\text { MUT }\end{array}$ & . 049 & $\begin{array}{l}.056 \\
.055 \\
.055\end{array}$ & & $\begin{array}{l}.104 \\
.145 \\
.145\end{array}$ & .136 & $\begin{array}{l}.170 \\
.301 \\
.301\end{array}$ & & $\begin{array}{l}.236 \\
.479 \\
.479\end{array}$ & .268 & .6 \\
\hline $\begin{array}{l}.50 \\
.50 \\
.50\end{array}$ & $\begin{array}{l}\text { MM } \\
\text { MU } \\
\text { MUT }\end{array}$ & $\begin{array}{l}.049 \\
.038 \\
.038\end{array}$ & $\begin{array}{l}.057 \\
.050 \\
.050\end{array}$ & & $\begin{array}{l}.105 \\
.14 \\
.14\end{array}$ & 142 & & & & & \\
\hline $\begin{array}{l}.60 \\
.60 \\
.60\end{array}$ & $\begin{array}{l}\text { MM } \\
\text { MU } \\
\text { MUT }\end{array}$ & $\begin{array}{l}.050 \\
.035 \\
.035\end{array}$ & & & $\begin{array}{l}.1 \\
.1 \\
.1\end{array}$ & & & & & .30 & \\
\hline $\begin{array}{l}.70 \\
.70 \\
.70\end{array}$ & $\begin{array}{l}\text { MM } \\
\text { MU } \\
\text { MUT }\end{array}$ & $\begin{array}{l}.050 \\
.030 \\
.030\end{array}$ & $\begin{array}{l}.061 \\
.044 \\
.044\end{array}$ & $\begin{array}{l}.091 \\
.084 \\
.084\end{array}$ & $\begin{array}{l}.130 \\
.147 \\
.147\end{array}$ & .227 & & $\begin{array}{l}.256 \\
.414 \\
.414\end{array}$ & $\begin{array}{l}.299 \\
.508 \\
.508\end{array}$ & $\begin{array}{l}.339 \\
.596 \\
.596\end{array}$ & .67 \\
\hline $\begin{array}{l}.80 \\
.80 \\
.80\end{array}$ & $\begin{array}{l}\text { MM } \\
\text { MU } \\
\text { MUT }\end{array}$ & $\begin{array}{l}.02 \\
.02\end{array}$ & $\begin{array}{l}.040 \\
.040\end{array}$ & $\begin{array}{l}.10 \\
.08 \\
.08\end{array}$ & $\begin{array}{l}.15 \\
.15 \\
.15\end{array}$ & $\begin{array}{l}.241 \\
.241\end{array}$ & $\begin{array}{r}.337 \\
.337\end{array}$ & $\begin{array}{l}.30 \\
.43 \\
.43\end{array}$ & $\begin{array}{l}.354 \\
.527 \\
.527\end{array}$ & $\begin{array}{l}.400 \\
.611 \\
.611\end{array}$ & $\begin{array}{l}68 \\
68\end{array}$ \\
\hline $\begin{array}{l}.90 \\
.90 \\
.90\end{array}$ & $\begin{array}{l}\text { MM } \\
\text { MU } \\
\text { MUT }\end{array}$ & .017 & $\begin{array}{l}.038 \\
.038\end{array}$ & $\begin{array}{l}.096 \\
.096\end{array}$ & $\begin{array}{l}.181 \\
.181\end{array}$ & .280 & & $\begin{array}{l}.476 \\
.476\end{array}$ & $\begin{array}{l}.561 \\
.561\end{array}$ & $\begin{array}{l}.636 \\
.636\end{array}$ & \\
\hline
\end{tabular}


POWER FOR 3 PAIRS

\begin{tabular}{|c|c|c|c|c|c|c|c|c|c|c|c|}
\hline \multicolumn{2}{|c|}{$\rho_{x y}$ METHOD } & .0 & .50 & 1.00 & 1.50 & 2.00 & 2.50 & 3.00 & 3.50 & 4.00 & 4.50 \\
\hline $\begin{array}{l}.00 \\
.00 \\
.00 \\
.00\end{array}$ & $\begin{array}{l}\text { MM } \\
\text { MU } \\
\text { MUT } \\
\text { UU }\end{array}$ & $\begin{array}{r}.049 \\
.050 \\
.045 \\
.050\end{array}$ & $\begin{array}{l}.068 \\
.077 \\
.063 \\
.077\end{array}$ & $\begin{array}{l}.119 \\
.160 \\
.115 \\
.160\end{array}$ & $\begin{array}{l}.196 \\
.296 \\
.201 \\
.296\end{array}$ & $\begin{array}{l}.294 \\
.165 \\
.320 \\
.465\end{array}$ & $\begin{array}{l}.400 \\
.638 \\
.462 \\
.638\end{array}$ & $\begin{array}{l}.509 \\
.782 \\
.606 \\
.782\end{array}$ & & $\begin{array}{l}.707 \\
.948 \\
.835 \\
.948\end{array}$ & $\begin{array}{l}.785 \\
.979 \\
.908 \\
.979\end{array}$ \\
\hline $\begin{array}{l}10 \\
.10 \\
10\end{array}$ & $\begin{array}{l}\text { MM } \\
\text { MU } \\
\text { MUT }\end{array}$ & $\begin{array}{r}.051 \\
.050 \\
.046\end{array}$ & $\begin{array}{r}.069 \\
.078 \\
.063\end{array}$ & $\begin{array}{l}.118 \\
.159 \\
.115\end{array}$ & $\begin{array}{r}198 \\
.294 \\
.201\end{array}$ & $\begin{array}{r}.295 \\
.463 \\
.321\end{array}$ & $\begin{array}{r}.401 \\
.636 \\
.461\end{array}$ & $\begin{array}{r}.512 \\
.783 \\
.605\end{array}$ & $\begin{array}{l}.61 \\
.88 \\
.73\end{array}$ & $\begin{array}{l}.708 \\
.949 \\
.836\end{array}$ & $\begin{array}{r}.787 \\
.979 \\
.909\end{array}$ \\
\hline $\begin{array}{l}20 \\
.20 \\
.20\end{array}$ & $\begin{array}{l}\text { MM } \\
\text { MU } \\
\text { MUT }\end{array}$ & $\begin{array}{r}.050 \\
.047 \\
.044\end{array}$ & $\begin{array}{l}.068 \\
.074 \\
.062\end{array}$ & $\begin{array}{r}.120 \\
.156 \\
.114\end{array}$ & $\begin{array}{l}201 \\
.291 \\
.201\end{array}$ & $\begin{array}{r}.300 \\
.462 \\
.322\end{array}$ & $\begin{array}{l}.409 \\
.637 \\
.462\end{array}$ & $\begin{array}{r}.519 \\
.786 \\
.607\end{array}$ & .62 & .73 & $\begin{array}{r}.794 \\
.981 \\
.912\end{array}$ \\
\hline $\begin{array}{l}30 \\
.30 \\
.30\end{array}$ & $\begin{array}{l}\text { MM } \\
\text { MU } \\
\text { MUT }\end{array}$ & $\begin{array}{r}.050 \\
.044 \\
.045\end{array}$ & $\begin{array}{l}.069 \\
.070 \\
.062\end{array}$ & $\begin{array}{r}.124 \\
.154 \\
.115\end{array}$ & $\begin{array}{l}207 \\
.291 \\
.206\end{array}$ & .314 & .426 & $\begin{array}{l}9 \\
9 \\
3\end{array}$ & & .737 & $\begin{array}{r}.814 \\
.981 \\
.914\end{array}$ \\
\hline $\begin{array}{r}40 \\
.40 \\
.40\end{array}$ & $\begin{array}{l}\text { MM } \\
\text { MU } \\
\text { MUT }\end{array}$ & $\begin{array}{l}.051 \\
.040 \\
.044\end{array}$ & $\begin{array}{l}.07 \\
.06 \\
.06\end{array}$ & $\begin{array}{l}.128 \\
.150 \\
.117\end{array}$ & $\begin{array}{l}219 \\
.286 \\
.209\end{array}$ & 320 & & & & & $\begin{array}{r}.837 \\
.983 \\
.918\end{array}$ \\
\hline .50 & $\begin{array}{l}\text { MM } \\
\text { MU } \\
\text { MUT }\end{array}$ & $\begin{array}{l}.051 \\
.034 \\
.045\end{array}$ & $\begin{array}{l}.07 \\
.05 \\
.06\end{array}$ & $\begin{array}{r}137 \\
.140 \\
.119\end{array}$ & $\begin{array}{r}235 \\
.282 \\
.214\end{array}$ & 356 & $\begin{array}{r}.482 \\
.648 \\
.489\end{array}$ & $\begin{array}{r}.605 \\
.798 \\
.636\end{array}$ & & .8 & $\begin{array}{r}.870 \\
.983 \\
.925\end{array}$ \\
\hline $\begin{array}{l}.60 \\
.60 \\
.60\end{array}$ & $\begin{array}{l}\text { MM } \\
\text { MU } \\
\text { MUT }\end{array}$ & $\begin{array}{l}.050 \\
.029 \\
.046\end{array}$ & $\begin{array}{r}.078 \\
.054 \\
.067\end{array}$ & $\begin{array}{l}.15 \\
.13 \\
.13\end{array}$ & $\begin{array}{r}.26 \\
.28 \\
.23\end{array}$ & 30. & $\begin{array}{l}4 \\
6 \\
2\end{array}$ & & .78 & $\begin{array}{r}.848 \\
.958 \\
.872\end{array}$ & $\begin{array}{r}.907 \\
.984 \\
.930\end{array}$ \\
\hline $\begin{array}{l}.70 \\
.70 \\
.70\end{array}$ & $\begin{array}{l}\text { MM } \\
\text { MU } \\
\text { MUT }\end{array}$ & $\begin{array}{l}.051 \\
.021 \\
.047\end{array}$ & $\begin{array}{l}.082 \\
.047 \\
.073\end{array}$ & $\begin{array}{l}.176 \\
.133 \\
.148\end{array}$ & $\begin{array}{l}.312 \\
.285 \\
.257\end{array}$ & $\begin{array}{l}.4 \\
.4\end{array}$ & $\begin{array}{l}.6 \\
.6 \\
.5\end{array}$ & $\begin{array}{l}0 \\
5 \\
1\end{array}$ & $\begin{array}{r}.837 \\
.909 \\
.805\end{array}$ & $\begin{array}{r}.905 \\
.960 \\
.887\end{array}$ & $\begin{array}{r}.948 \\
.984 \\
.939\end{array}$ \\
\hline $\begin{array}{l}.80 \\
.80 \\
.80\end{array}$ & $\begin{array}{l}\text { MM } \\
\text { MU } \\
\text { MUT }\end{array}$ & $\begin{array}{l}.049 \\
.014 \\
.049\end{array}$ & $\begin{array}{l}.05 \\
.03 \\
.08\end{array}$ & $\begin{array}{l}.224 \\
.130 \\
.183\end{array}$ & $\begin{array}{l}.398 \\
.294 \\
.312\end{array}$ & $\begin{array}{l}.5 \\
.4 \\
.4\end{array}$ & $\begin{array}{l}.733 \\
.681 \\
.610\end{array}$ & $\begin{array}{r}.8 \\
.8 \\
.7\end{array}$ & $\begin{array}{r}.921 \\
.911 \\
.837\end{array}$ & $\begin{array}{r}.963 \\
.960 \\
.905\end{array}$ & $\begin{array}{r}.984 \\
.984 \\
.949\end{array}$ \\
\hline $\begin{array}{r}90 \\
.90\end{array}$ & $\begin{array}{l}\text { MM } \\
\text { MU } \\
\text { MUT }\end{array}$ & $\begin{array}{l}.050 \\
.007 \\
.056\end{array}$ & $\begin{array}{l}.1 \\
.0 \\
.1\end{array}$ & $\begin{array}{l}3 \\
.1 \\
2\end{array}$ & .6 & $\begin{array}{l}.79 \\
.51 \\
.59\end{array}$ & $\begin{array}{r}.917 \\
.694 \\
.722\end{array}$ & $\begin{array}{r}.970 \\
.826 \\
.818\end{array}$ & $\begin{array}{l}.992 \\
.910 \\
.889\end{array}$ & $\begin{array}{r}.998 \\
.958 \\
.938\end{array}$ & $\begin{array}{r}1.000 \\
.982 \\
.966\end{array}$ \\
\hline
\end{tabular}


POWER FOR 4 PAIRS

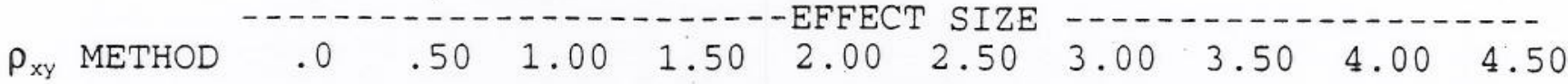

$\begin{array}{llllllllllll}.00 & \text { MM } & .050 & .080 & .171 & .314 & .487 & .657 & .796 & .892 & .950 & .980\end{array}$

$\begin{array}{llllllllllll}.00 & \text { MU } & .049 & .092 & .223 & .426 & .654 & .835 & .940 & .983 & .996 & .999\end{array}$

$\begin{array}{llllllllllll}.00 & \text { MUT } & .046 & .078 & .179 & .351 & .559 & .754 & .891 & .961 & .989 & .997\end{array}$

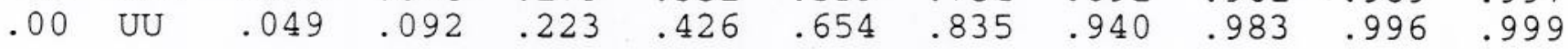

$\begin{array}{llllllllllll}.10 & \text { MM } & .050 & .081 & .173 & .317 & .492 & .661 & .798 & .893 & .949 & .979\end{array}$

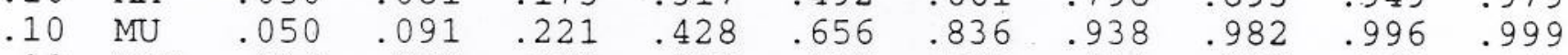

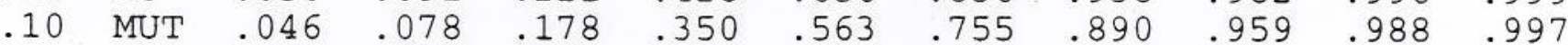

$\begin{array}{llllllllllll}.20 & \text { MM } & .050 & .083 & .179 & .329 & .507 & .676 & .812 & .904 & .956 & .982\end{array}$

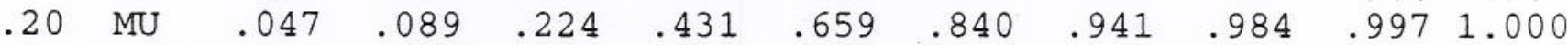

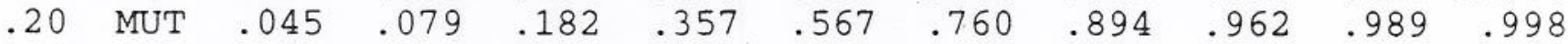

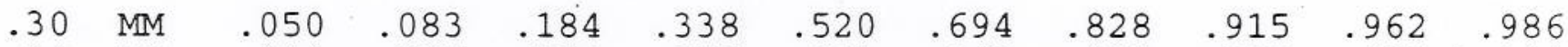

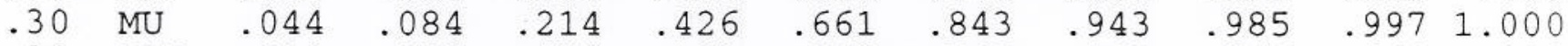

$\begin{array}{llllllllllll}.30 & \text { MUT } & .044 & .076 & .179 & .353 & .568 & .765 & .897 & .964 & .990 & .998\end{array}$

$\begin{array}{llllllllllll}.40 & \text { MM } & .050 & .087 & .196 & .362 & .553 & .727 & .856 & .933 & .973 & .991\end{array}$

$\begin{array}{llllllllllll}.40 & \text { MU } & .037 & .079 & .210 & .429 & .669 & .852 & .948 & .987 & .997 & 1.000\end{array}$

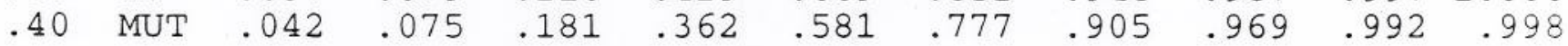

$\begin{array}{llllllllllll}.50 & \text { MM } & .050 & .090 & .212 & .396 & .598 & .771 & .888 & .953 & .983 & .995\end{array}$

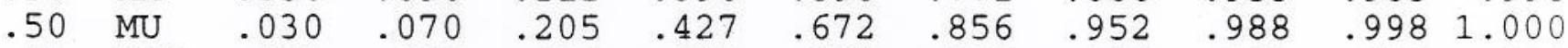



$\begin{array}{llllllllllll}.60 & \mathrm{MM} & .050 & .097 & .240 & .447 & .658 & .823 & .924 & .973 & .992 & .998\end{array}$

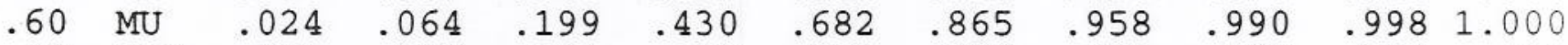

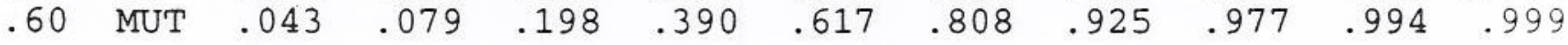

$\begin{array}{llllllllllll}.70 & \text { MM } & .051 & .110 & .283 & .525 & .747 & .893 & .964 & .990 & .998 & 1.000\end{array}$

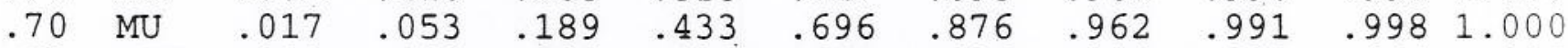



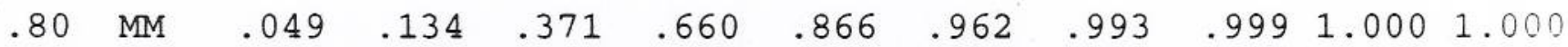

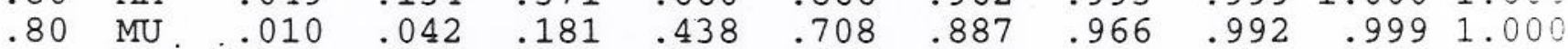

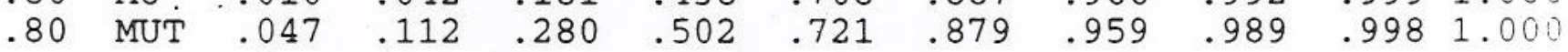

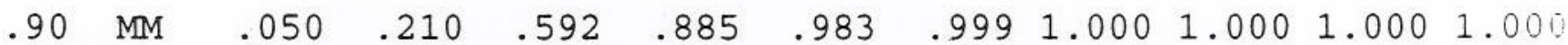

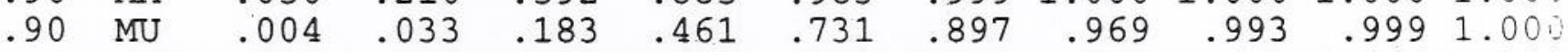

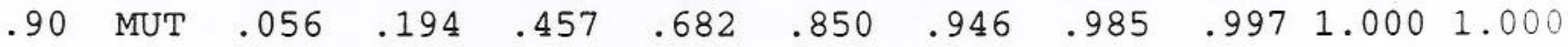


POWER FOR 5 PAIRS

\begin{tabular}{|c|c|c|c|c|c|c|c|c|c|c|c|}
\hline xy 1 & HO & .0 & .50 & 1.00 & .50 & & 2.50 & 3.00 & 3.50 & 4.00 & 0 \\
\hline $\begin{array}{l}.00 \\
.00 \\
.00 \\
.00\end{array}$ & $\begin{array}{l}\text { MM } \\
\text { MU } \\
\text { MUT } \\
\text { UU }\end{array}$ & $\begin{array}{l}.051 \\
.052 \\
.047 \\
.052\end{array}$ & $\begin{array}{l}.095 \\
.108 \\
.095 \\
.108\end{array}$ & $\begin{array}{l}.231 \\
.286 \\
.247 \\
.286\end{array}$ & $\begin{array}{l}.439 \\
.548 \\
.486 \\
.548\end{array}$ & $\begin{array}{l}.660 \\
.788 \\
.731 \\
.788\end{array}$ & $\begin{array}{l}.832 \\
.931 \\
.899 \\
.931\end{array}$ & & $\begin{array}{l}.980 \\
.997 \\
.995 \\
.997\end{array}$ & $\begin{array}{r}.995 \\
1.000 \\
.999 \\
1.000\end{array}$ & $\begin{array}{r}.9 \\
1.0 \\
1.0 \\
1.0\end{array}$ \\
\hline $\begin{array}{l}.10 \\
.10 \\
.10\end{array}$ & $\begin{array}{l}\text { MM } \\
\text { MU } \\
\text { MUT }\end{array}$ & $\begin{array}{l}.048 \\
.048 \\
.045\end{array}$ & $\begin{array}{l}.095 \\
.105 \\
.093\end{array}$ & $\begin{array}{l}.234 \\
.286 \\
.246\end{array}$ & $\begin{array}{l}.446 \\
.550 \\
.488\end{array}$ & $\begin{array}{r}.666 \\
.793 \\
.736\end{array}$ & $\begin{array}{r}.839 \\
.934 \\
.902\end{array}$ & & $\begin{array}{l}.982 \\
.998 \\
.995\end{array}$ & $\begin{array}{r}.996 \\
1.000 \\
.999\end{array}$ & $\begin{array}{r}.9 \\
1.0 \\
1.0\end{array}$ \\
\hline $\begin{array}{l}.20 \\
.20 \\
.20\end{array}$ & $\begin{array}{l}\text { MM } \\
\text { MU } \\
\text { MUT }\end{array}$ & $\begin{array}{l}.050 \\
.046 \\
.045\end{array}$ & $\begin{array}{l}.098 \\
.104 \\
.094\end{array}$ & $\begin{array}{l}.238 \\
.281 \\
.247\end{array}$ & $\begin{array}{r}.454 \\
.548 \\
.488\end{array}$ & $\begin{array}{l}.679 \\
.794 \\
.737\end{array}$ & $\begin{array}{l}.848 \\
.935 \\
.904\end{array}$ & & $\begin{array}{r}.984 \\
.998 \\
.996\end{array}$ & $\begin{array}{r}.996 \\
1.000 \\
.999\end{array}$ & $\begin{array}{r}.999 \\
1.000 \\
1.000\end{array}$ \\
\hline $\begin{array}{l}.30 \\
.30 \\
.30\end{array}$ & $\begin{array}{l}\text { MM } \\
\text { MU } \\
\text { MUT }\end{array}$ & $\begin{array}{l}.050 \\
.042 \\
.044\end{array}$ & $\begin{array}{l}.09 \\
.09 \\
.09\end{array}$ & $\begin{array}{l}.24 \\
.27 \\
.24\end{array}$ & .47 & .699 & $\begin{array}{l}.865 \\
.938 \\
.908\end{array}$ & $\begin{array}{l}.9 \\
.9 \\
.9\end{array}$ & $\begin{array}{l}.987 \\
.998 \\
.996\end{array}$ & $\begin{array}{r}.997 \\
1.000 \\
1.000\end{array}$ & $\begin{array}{l}1.000 \\
1.000 \\
1.000\end{array}$ \\
\hline $\begin{array}{l}.40 \\
.40 \\
.40\end{array}$ & $\begin{array}{l}\text { MM } \\
\text { MU } \\
\text { MUT }\end{array}$ & $\begin{array}{l}.050 \\
.036 \\
.042\end{array}$ & $\begin{array}{l}10 \\
.09 \\
.09\end{array}$ & $\begin{array}{l}.266 \\
.273 \\
.249\end{array}$ & 50 & .73 & $\begin{array}{r}.8 \\
.9 \\
.9\end{array}$ & .96 & $\begin{array}{l}.991 \\
.999 \\
.997\end{array}$ & $\begin{array}{r}.998 \\
1.000 \\
1.000\end{array}$ & $\begin{array}{l}1.000 \\
1.000 \\
1.000\end{array}$ \\
\hline $\begin{array}{l}.50 \\
.50 \\
.50\end{array}$ & $\begin{array}{l}\text { MM } \\
\text { MU } \\
\text { MUT }\end{array}$ & $\begin{array}{l}.050 \\
.030 \\
.041\end{array}$ & $\begin{array}{l}.11 \\
.08 \\
.09\end{array}$ & $\begin{array}{l}.29 \\
.26 \\
.25\end{array}$ & $\begin{array}{l}.5 \\
.5 \\
.5\end{array}$ & $.7 \xi$ & 9 & 978 & $\begin{array}{l}.995 \\
.999 \\
.998\end{array}$ & $\begin{array}{r}.999 \\
1.000 \\
1.000\end{array}$ & $\begin{array}{l}1.000 \\
1.000 \\
1.000\end{array}$ \\
\hline $\begin{array}{l}.60 \\
.60 \\
.60\end{array}$ & $\begin{array}{l}\text { MM } \\
\text { MU } \\
\text { MUT }\end{array}$ & $\begin{array}{l}.051 \\
.022 \\
.041\end{array}$ & $\begin{array}{l}.119 \\
.074 \\
.095\end{array}$ & $\begin{array}{l}.32 \\
.26 \\
.27\end{array}$ & $\begin{array}{l}.6 \\
.5 \\
.5\end{array}$ & .834 & .9 & $\begin{array}{r}.990 \\
.993 \\
.989\end{array}$ & $\begin{array}{r}.999 \\
.999 \\
.999\end{array}$ & $\begin{array}{l}1.000 \\
1.000 \\
1.000\end{array}$ & $\begin{array}{l}1.000 \\
1.000 \\
1.000\end{array}$ \\
\hline $\begin{array}{l}.70 \\
.70 \\
.70\end{array}$ & $\begin{array}{l}\text { MM } \\
\text { MU } \\
\text { MUT }\end{array}$ & $\begin{array}{l}.049 \\
.014 \\
.043\end{array}$ & $\begin{array}{l}.138 \\
.062 \\
.110\end{array}$ & $\begin{array}{l}.39 \\
.25 \\
.30\end{array}$ & .59 & $\begin{array}{r}.90 \\
.84 \\
.83\end{array}$ & $\begin{array}{l}.980 \\
.964 \\
.957\end{array}$ & $\begin{array}{r}.997 \\
.995 \\
.993\end{array}$ & $\begin{array}{r}1.000 \\
.999 \\
.999\end{array}$ & $\begin{array}{l}1.000 \\
1.000 \\
1.000\end{array}$ & $\begin{array}{l}1.000 \\
1.000 \\
1.000\end{array}$ \\
\hline $\begin{array}{l}.80 \\
.80 \\
.80\end{array}$ & $\begin{array}{l}\text { MM } \\
\text { MU } \\
\text { MUT }\end{array}$ & $\begin{array}{l}.049 \\
.007 \\
.047\end{array}$ & $\begin{array}{l}.176 \\
.049 \\
.147\end{array}$ & $\begin{array}{l}.51 \\
.24 \\
.40\end{array}$ & $\begin{array}{l}.83 \\
.58 \\
.68\end{array}$ & $\begin{array}{l}.971 \\
.857 \\
.893\end{array}$ & $\begin{array}{r}.997 \\
.971 \\
.978\end{array}$ & $\begin{array}{r}1.000 \\
.996 \\
.997\end{array}$ & $\begin{array}{l}1.000 \\
1.000 \\
1.000\end{array}$ & $\begin{array}{l}1.000 \\
1.000 \\
1.000\end{array}$ & $\begin{array}{l}1.000 \\
1.000 \\
1.000\end{array}$ \\
\hline $\begin{array}{l}.90 \\
.90 \\
.90\end{array}$ & $\begin{array}{l}\text { MM } \\
\text { MU } \\
\text { MUT }\end{array}$ & $\begin{array}{l}.049 \\
.002 \\
.056\end{array}$ & $\begin{array}{l}.285 \\
.035 \\
.272\end{array}$ & $\begin{array}{l}.77 \\
.23 \\
.64\end{array}$ & $\begin{array}{r}.976 \\
.604 \\
.869\end{array}$ & $\begin{array}{l}.999 \\
.874 \\
.972\end{array}$ & $\begin{array}{r}1.000 \\
.976 \\
.997\end{array}$ & $\begin{array}{r}1.000 \\
.997 \\
1.000\end{array}$ & $\begin{array}{l}1.000 \\
1.000 \\
1.000\end{array}$ & $\begin{array}{l}1.000 \\
1.000 \\
1.000\end{array}$ & $\begin{array}{l}1.00 \\
1.00 \\
1.00\end{array}$ \\
\hline
\end{tabular}




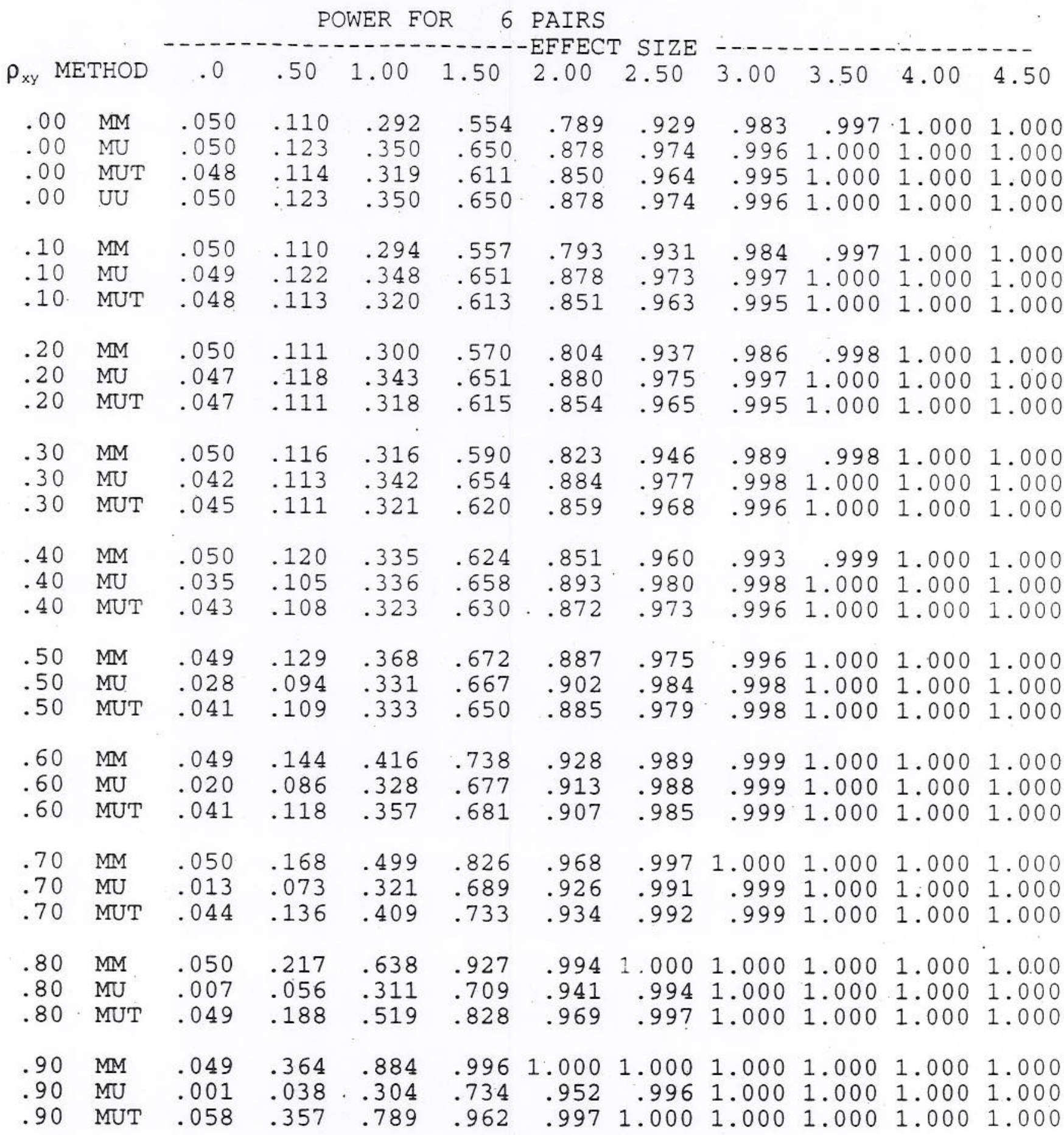


POWER FOR 7 PAIRS

\begin{tabular}{|c|c|c|c|c|c|c|c|c|c|c|c|}
\hline$D_{x y} 1$ & $O D$ & .0 & .50 & 1.00 & 1.50 & 2.00 & 2.50 & 3.00 & 3.50 & .00 & 1.50 \\
\hline $\begin{array}{l}.00 \\
.00 \\
.00 \\
.00\end{array}$ & $\begin{array}{l}\text { MM } \\
\text { MU } \\
\text { MUT } \\
\text { UU }\end{array}$ & $\begin{array}{l}.050 \\
.049 \\
.046 \\
.049\end{array}$ & $\begin{array}{l}.123 \\
.139 \\
.123 \\
.139\end{array}$ & $\begin{array}{l}.349 \\
.401 \\
.363 \\
.401\end{array}$ & $\begin{array}{l}.646 \\
.729 \\
.686 \\
.729\end{array}$ & $\begin{array}{r}.874 \\
.929 \\
.908 \\
.929\end{array}$ & $\begin{array}{l}.971 \\
.990 \\
.985 \\
.990\end{array}$ & $\begin{array}{l}.996 \\
.999 \\
.999 \\
.999\end{array}$ & $\begin{array}{l}1.000 \\
1.000 \\
1.000 \\
1.000\end{array}$ & $\begin{array}{l}1.000 \\
1.000 \\
1.000 \\
1.000\end{array}$ & $\begin{array}{l}1.000 \\
1.000 \\
1.000 \\
1.000\end{array}$ \\
\hline $\begin{array}{l}.10 \\
.10 \\
.10\end{array}$ & $\begin{array}{l}\text { MM } \\
\text { MU } \\
\text { MUT }\end{array}$ & $\begin{array}{l}.050 \\
.048 \\
.045\end{array}$ & $\begin{array}{l}.125 \\
.137 \\
.122\end{array}$ & $\begin{array}{l}.353 \\
.403 \\
.365\end{array}$ & $\begin{array}{l}.652 \\
.730 \\
.688\end{array}$ & $\begin{array}{l}.876 \\
.930 \\
.909\end{array}$ & $\begin{array}{l}.972 \\
.990 \\
.985\end{array}$ & & & & $\begin{array}{l}1.000 \\
1.000 \\
1.000\end{array}$ \\
\hline $\begin{array}{l}.20 \\
.20 \\
.20\end{array}$ & $\begin{array}{l}\text { MM } \\
\text { MU } \\
\text { MUT }\end{array}$ & $\begin{array}{l}.051 \\
.046 \\
.045\end{array}$ & $\begin{array}{l}.125 \\
.132 \\
.120\end{array}$ & $\begin{array}{l}.361 \\
.402 \\
.365\end{array}$ & $\begin{array}{l}.665 \\
.733 \\
.692\end{array}$ & $\begin{array}{l}.885 \\
.931 \\
.911\end{array}$ & $\begin{array}{l}.975 \\
.990 \\
.985\end{array}$ & $\begin{array}{r}.996 \\
.999 \\
.999\end{array}$ & & & $\begin{array}{l}1.000 \\
1.000 \\
1.000\end{array}$ \\
\hline $\begin{array}{l}.30 \\
.30 \\
.30\end{array}$ & $\begin{array}{l}\text { MM } \\
\text { MU } \\
\text { MUT }\end{array}$ & $\begin{array}{l}.051 \\
.042 \\
.043\end{array}$ & $\begin{array}{l}.131 \\
.127 \\
.119\end{array}$ & $\begin{array}{l}.374 \\
.399 \\
.367\end{array}$ & $\begin{array}{l}.688 \\
.737 \\
.699\end{array}$ & $\begin{array}{l}.901 \\
.937 \\
.918\end{array}$ & $\begin{array}{l}.981 \\
.991 \\
.987\end{array}$ & $\begin{array}{r}.998 \\
.999 \\
.999\end{array}$ & $\begin{array}{l}1.000 \\
1.000 \\
1.000\end{array}$ & $\begin{array}{l}1.000 \\
1.000 \\
1.000\end{array}$ & $\begin{array}{l}1.000 \\
1.000 \\
1.000\end{array}$ \\
\hline $\begin{array}{l}.40 \\
.40 \\
.40\end{array}$ & $\begin{array}{l}\text { MM } \\
\text { MU } \\
\text { MUT }\end{array}$ & $\begin{array}{l}.049 \\
.035 \\
.039\end{array}$ & $\begin{array}{l}.138 \\
.122 \\
.119\end{array}$ & $\begin{array}{l}.404 \\
.399 \\
.377\end{array}$ & $\begin{array}{l}.724 \\
.746 \\
.714\end{array}$ & $\begin{array}{l}.922 \\
.944 \\
.927\end{array}$ & $\begin{array}{l}.987 \\
.993 \\
.990\end{array}$ & $\begin{array}{r}.998 \\
1.000 \\
.999\end{array}$ & & & $\begin{array}{l}1.000 \\
1.000 \\
1.000\end{array}$ \\
\hline $\begin{array}{l}.50 \\
.50 \\
.50\end{array}$ & $\begin{array}{l}\text { MM } \\
\text { MU } \\
\text { MUT }\end{array}$ & $\begin{array}{l}.050 \\
.028 \\
.038\end{array}$ & $\begin{array}{l}.152 \\
.113 \\
.123\end{array}$ & $\begin{array}{r}.444 \\
.398 \\
.392\end{array}$ & $\begin{array}{r}.770 \\
.759 \\
.735\end{array}$ & $\begin{array}{l}.947 \\
.951 \\
.939\end{array}$ & $\begin{array}{l}.993 \\
.995 \\
.993\end{array}$ & $\begin{array}{r}.999 \\
1.000 \\
1.000\end{array}$ & $\begin{array}{l}1.000 \\
1.000 \\
1.000\end{array}$ & $\begin{array}{l}1.000 \\
1.000 \\
1.000\end{array}$ & $\begin{array}{l}1.000 \\
1.000 \\
1.000\end{array}$ \\
\hline $\begin{array}{l}.60 \\
.60 \\
.60\end{array}$ & $\begin{array}{l}\text { MM } \\
\text { MU } \\
\text { MUT }\end{array}$ & $\begin{array}{l}.049 \\
.021 \\
.038\end{array}$ & $\begin{array}{l}.167 \\
.097 \\
.128\end{array}$ & $\begin{array}{l}.49 \\
.39 \\
.41\end{array}$ & $\begin{array}{l}.83 \\
.76 \\
.76\end{array}$ & $\begin{array}{l}.971 \\
.958 \\
.953\end{array}$ & $\begin{array}{l}.998 \\
.997 \\
.996\end{array}$ & $\begin{array}{l}1.000 \\
1.000 \\
1.000\end{array}$ & & $\begin{array}{l}1.000 \\
1.000 \\
1.000\end{array}$ & $\begin{array}{l}1.00 \\
1.00 \\
1.00\end{array}$ \\
\hline $\begin{array}{l}.70 \\
.70 \\
.70\end{array}$ & $\begin{array}{l}\text { MM } \\
\text { MU } \\
\text { MUT }\end{array}$ & $\begin{array}{l}.049 \\
.012 \\
.041\end{array}$ & $\begin{array}{l}.200 \\
.083 \\
.154\end{array}$ & $\begin{array}{l}.593 \\
.388 \\
.478\end{array}$ & $\begin{array}{r}.903 \\
.789 \\
.823\end{array}$ & $\begin{array}{l}.990 \\
.968 \\
.973\end{array}$ & $\begin{array}{r}1.000 \\
.998 \\
.998\end{array}$ & $\begin{array}{l}1.000 \\
1.000 \\
1.000\end{array}$ & & & $\begin{array}{l}1.00 \\
1.00 \\
1.00\end{array}$ \\
\hline $\begin{array}{l}.80 \\
.80 \\
.80\end{array}$ & $\begin{array}{l}\text { MM } \\
\text { MU } \\
\text { MUT }\end{array}$ & $\begin{array}{l}.049 \\
.005 \\
.047\end{array}$ & $\begin{array}{l}.064 \\
.219\end{array}$ & $\begin{array}{l}.737 \\
.382 \\
.612\end{array}$ & $\begin{array}{l}.971 \\
.810 \\
.906\end{array}$ & $\begin{array}{r}.999 \\
.977 \\
.991\end{array}$ & $\begin{array}{l}.000 \\
.999 \\
.000\end{array}$ & $\begin{array}{l}1.000 \\
1.000 \\
1.000\end{array}$ & $\begin{array}{l}1.000 \\
1.000 \\
1.000\end{array}$ & $\begin{array}{l}1.000 \\
1.000 \\
1.000\end{array}$ & $\begin{array}{l}1.0 \\
1.0 \\
1.0\end{array}$ \\
\hline 90 & $\begin{array}{l}\text { MM } \\
\text { MU } \\
\text { MUT }\end{array}$ & $\begin{array}{l}.050 \\
.001 \\
.055\end{array}$ & $\begin{array}{l}.042 \\
.422\end{array}$ & $\begin{array}{l}.944 \\
.381 \\
.879\end{array}$ & $\begin{array}{r}1.000 \\
.839 \\
.989\end{array}$ & $\begin{array}{r}.986 \\
1.000\end{array}$ & $\begin{array}{l}1.000 \\
1.000\end{array}$ & $\begin{array}{l}1.000 \\
1.000 \\
1.000\end{array}$ & $\begin{array}{l}1.000 \\
1.000 \\
1.000\end{array}$ & $\begin{array}{l}1.000 \\
1.000 \\
1.000\end{array}$ & \\
\hline
\end{tabular}




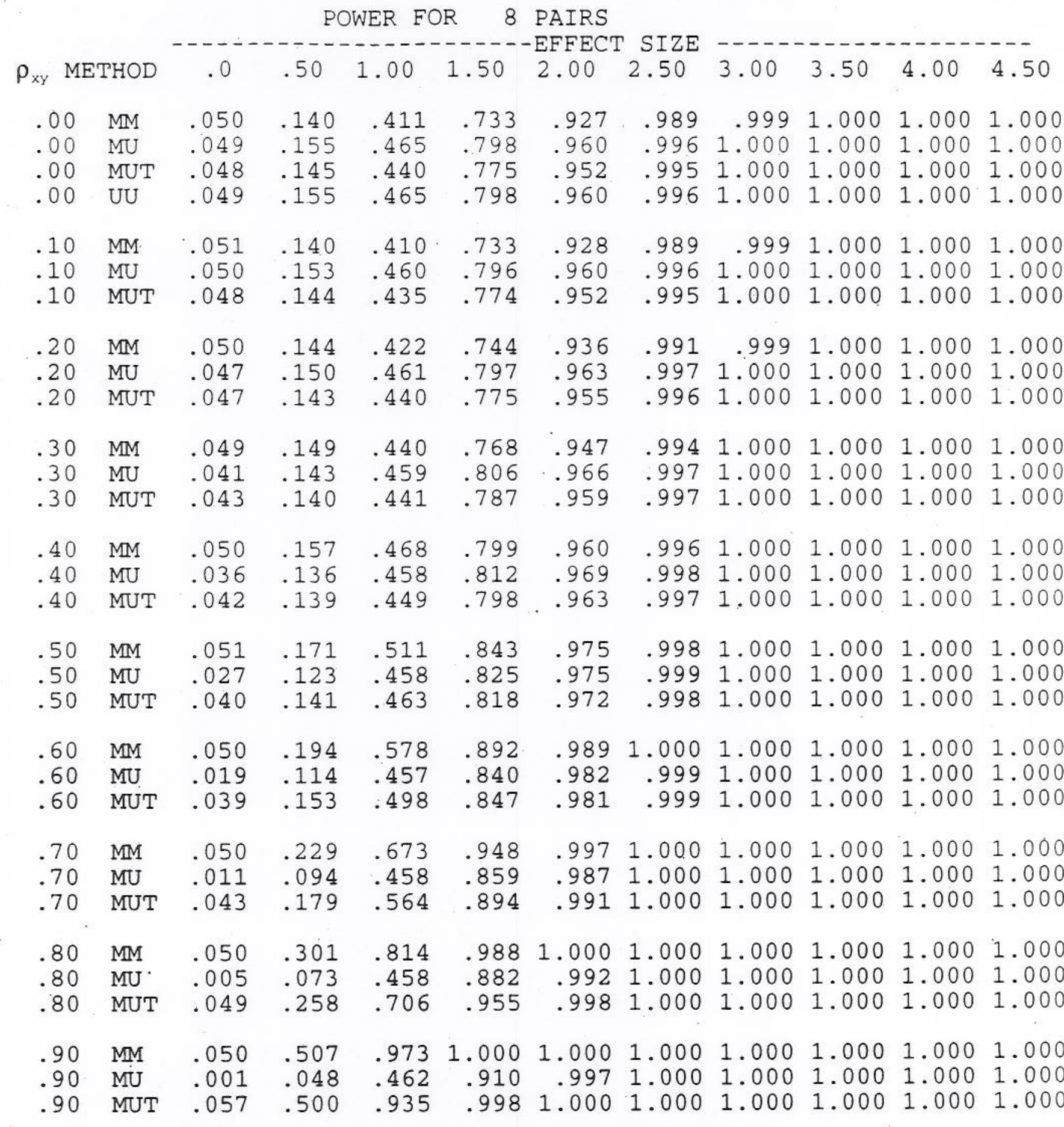


POWER FOR 9 PAIRS

\begin{tabular}{|c|c|c|c|c|c|c|c|c|c|c|c|}
\hline$x_{Y} 1$ & $D D$ & .0 & .50 & 1.00 & 1.50 & 2. & 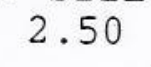 & 00 & 50 & 4.00 & 4.50 \\
\hline $\begin{array}{l}.00 \\
.00 \\
.00 \\
.00\end{array}$ & $\begin{array}{l}\text { MM } \\
\text { MU } \\
\text { MUT } \\
\text { UU }\end{array}$ & $\begin{array}{l}.051 \\
.051 \\
.050 \\
.051\end{array}$ & $\begin{array}{l}.158 \\
.172 \\
.164 \\
.172\end{array}$ & $\begin{array}{l}.465 \\
.515 \\
.496 \\
.515\end{array}$ & $\begin{array}{l}.796 \\
.847 \\
.831 \\
.847\end{array}$ & $\begin{array}{l}.959 \\
.978 \\
.974 \\
.978\end{array}$ & $\begin{array}{l}.996 \\
.999 \\
.998 \\
.999\end{array}$ & $\begin{array}{l}1.000 \\
1.000 \\
1.000 \\
1.000\end{array}$ & & & $\begin{array}{l}1 . \\
1 . \\
1 .\end{array}$ \\
\hline $\begin{array}{l}10 \\
10 \\
10\end{array}$ & $\begin{array}{l}\text { MM } \\
\text { MU } \\
\text { MUT }\end{array}$ & $\begin{array}{l}.050 \\
.050 \\
.048\end{array}$ & $\begin{array}{l}.156 \\
.169 \\
.161\end{array}$ & $\begin{array}{l}.463 \\
.512 \\
.492\end{array}$ & $\begin{array}{l}.800 \\
.849 \\
.833\end{array}$ & $\begin{array}{r}.960 \\
.979 \\
.975\end{array}$ & $\begin{array}{r}.996 \\
.999 \\
.998\end{array}$ & & & & $\begin{array}{l}1 \\
1\end{array}$ \\
\hline $\begin{array}{l}.20 \\
.20 \\
.20\end{array}$ & $\begin{array}{l}\text { MM } \\
\text { MU } \\
\text { MUT }\end{array}$ & $\begin{array}{l}.050 \\
.046 \\
.047\end{array}$ & $\begin{array}{l}.15 \\
.16 \\
.15\end{array}$ & $\begin{array}{r}.480 \\
.515 \\
.497\end{array}$ & $\begin{array}{l}.811 \\
.851 \\
.836\end{array}$ & $\begin{array}{r}.963 \\
.979 \\
.976\end{array}$ & $\begin{array}{r}996 \\
.999 \\
.998\end{array}$ & & & & $\begin{array}{l}1 \\
1\end{array}$ \\
\hline $\begin{array}{l}30 \\
.30 \\
.30\end{array}$ & $\begin{array}{l}\text { MM } \\
\text { MU } \\
\text { MUT }\end{array}$ & $\begin{array}{l}.050 \\
.042 \\
.045\end{array}$ & $\begin{array}{l}.16 \\
.16 \\
.15\end{array}$ & $\begin{array}{l}.498 \\
.516 \\
.501\end{array}$ & .832 & $\begin{array}{r}.972 \\
.982 \\
.979\end{array}$ & $\begin{array}{l}8 \\
9 \\
9\end{array}$ & & & & $\begin{array}{l}1.000 \\
1.000 \\
1.000\end{array}$ \\
\hline $\begin{array}{l}.40 \\
.40 \\
.40\end{array}$ & $\begin{array}{l}\text { MM } \\
\text { MU } \\
\text { MUT }\end{array}$ & $\begin{array}{l}.03 \\
.04\end{array}$ & $\begin{array}{l}.17 \\
.15 \\
.15\end{array}$ & .5 & .8 & $\begin{array}{l}.981 \\
.986 \\
.983\end{array}$ & $\begin{array}{l}.999 \\
.999 \\
.999\end{array}$ & & & & $\begin{array}{l}1.000 \\
1.000 \\
1.000\end{array}$ \\
\hline $\begin{array}{l}.50 \\
.50 \\
.50\end{array}$ & $\begin{array}{l}\text { MM } \\
\text { MU } \\
\text { MUT }\end{array}$ & .0 & $\begin{array}{l}.19 \\
.14 \\
.16\end{array}$ & .5 & $\begin{array}{l}.85 \\
.87 \\
.87\end{array}$ & .989 & $\begin{array}{l}1.000 \\
1.000 \\
1.000\end{array}$ & & & & $\begin{array}{l}1.000 \\
1.000 \\
1.000\end{array}$ \\
\hline $\begin{array}{l}.60 \\
.60 \\
.60\end{array}$ & $\begin{array}{l}\text { MM } \\
\text { MU } \\
\text { MUT }\end{array}$ & .03 & 17 & .56 & $\begin{array}{l}.935 \\
.891 \\
.902\end{array}$ & $\begin{array}{l}.996 \\
.992 \\
.993\end{array}$ & $\begin{array}{l}1.000 \\
1.000 \\
1.000\end{array}$ & $\begin{array}{l}1 . \\
1 . \\
1 .\end{array}$ & & & $\begin{array}{l}1.000 \\
1.000 \\
1.000\end{array}$ \\
\hline $\begin{array}{l}.70 \\
.70 \\
.70\end{array}$ & $\begin{array}{l}\text { MM } \\
\text { MU } \\
\text { MUT }\end{array}$ & $\begin{array}{l}.05 \\
.01 \\
.04\end{array}$ & $\begin{array}{l}.26 \\
.10 \\
.20\end{array}$ & $\begin{array}{l}.740 \\
.524 \\
.641\end{array}$ & $\begin{array}{r}.972 \\
.909 \\
.939\end{array}$ & $\begin{array}{r}.999 \\
.995 \\
.997\end{array}$ & $\begin{array}{l}1.000 \\
1.000 \\
1.000\end{array}$ & $\begin{array}{l}1.000 \\
1.000 \\
1.000\end{array}$ & $\begin{array}{l}1.000 \\
1.000 \\
1.000\end{array}$ & & $\begin{array}{l}1.000 \\
1.000 \\
1.000\end{array}$ \\
\hline $\begin{array}{l}.80 \\
.80 \\
.80\end{array}$ & $\begin{array}{l}\text { MM } \\
\text { MU . } \\
\text { MUT }\end{array}$ & $\begin{array}{l}.05 \\
.00 \\
.05\end{array}$ & $\begin{array}{l}.35 \\
.08 \\
.30\end{array}$ & $\begin{array}{l}.871 \\
.530 \\
.787\end{array}$ & $\begin{array}{l}.996 \\
.929 \\
.981\end{array}$ & $\begin{array}{r}1.000 \\
.998 \\
1.000\end{array}$ & $\begin{array}{l}1.000 \\
1.000 \\
1.000\end{array}$ & $\begin{array}{l}1.000 \\
1.000 \\
1.000\end{array}$ & $\begin{array}{l}1.000 \\
1.000 \\
1.000\end{array}$ & $\begin{array}{l}1.000 \\
1.000 \\
1.000\end{array}$ & $\begin{array}{l}1.000 \\
1.000 \\
1.000\end{array}$ \\
\hline $\begin{array}{l}.90 \\
.90 \\
.90\end{array}$ & $\begin{array}{l}\text { MM } \\
\text { MU } \\
\text { MUT }\end{array}$ & $\begin{array}{l}.000 \\
.058\end{array}$ & .05 & $\begin{array}{l}.988 \\
.539 \\
.969\end{array}$ & $\begin{array}{r}1.000 \\
.955 \\
1.000\end{array}$ & $\begin{array}{r}1.000 \\
.999 \\
1.000\end{array}$ & $\begin{array}{l}1.000 \\
1.000 \\
1.000\end{array}$ & $\begin{array}{l}1.000 \\
1.000 \\
1.000\end{array}$ & $\begin{array}{l}1.000 \\
1.000 \\
1.000\end{array}$ & $\begin{array}{l}1.000 \\
1.000 \\
1.000\end{array}$ & $\begin{array}{l}1.000 \\
1.000 \\
1.000\end{array}$ \\
\hline
\end{tabular}


POWER FOR 10 PAIRS

\begin{tabular}{|c|c|c|c|c|c|c|c|c|c|c|c|}
\hline \multicolumn{2}{|c|}{ METHOD } & .0 & .50 & 1.00 & .50 & 2.00 & 2.50 & 3.00 & 3.50 & 4.00 & .50 \\
\hline $\begin{array}{l}.00 \\
.00 \\
.00 \\
.00\end{array}$ & $\begin{array}{l}\text { MM } \\
\text { MU } \\
\text { MUT } \\
\text { UU }\end{array}$ & $\begin{array}{l}.049 \\
.049 \\
.046 \\
.049\end{array}$ & $\begin{array}{l}.169 \\
.183 \\
.171 \\
.183\end{array}$ & $\begin{array}{l}.514 \\
.561 \\
.534 \\
.561\end{array}$ & $\begin{array}{l}.847 \\
.888 \\
.871 \\
.888\end{array}$ & $\begin{array}{l}.977 \\
.988 \\
.985 \\
.988\end{array}$ & $\begin{array}{l}.998 \\
.999 \\
.999 \\
.999\end{array}$ & $\begin{array}{l}00 \\
00 \\
00 \\
00\end{array}$ & $\begin{array}{ll}0 & 0 \\
0 & 0 \\
0 & 0 \\
0 & 0\end{array}$ & $\begin{array}{ll}0 & 0 \\
0 & 0 \\
0 & 0 \\
0 & 0\end{array}$ & $\begin{array}{l}1 \\
1 \\
1 \\
1\end{array}$ \\
\hline $\begin{array}{l}10 \\
.10 \\
.10\end{array}$ & $\begin{array}{l}\text { MM } \\
\text { MU } \\
\text { MUT }\end{array}$ & $\begin{array}{l}.050 \\
.049 \\
.046\end{array}$ & $\begin{array}{l}.173 \\
.185 \\
.172\end{array}$ & $\begin{array}{r}518 \\
.562\end{array}$ & $\begin{array}{l}.8 \\
.8 \\
.87\end{array}$ & $\begin{array}{l}.978 \\
.989 \\
.986\end{array}$ & $\begin{array}{r}.999 \\
1.000 \\
.999\end{array}$ & & $\begin{array}{l}00 \\
00 \\
00\end{array}$ & & \\
\hline $\begin{array}{l}.20 \\
.20 \\
.20\end{array}$ & $\begin{array}{l}\text { MM } \\
\text { MU } \\
\text { MUT }\end{array}$ & $\begin{array}{l}.05 \\
.04 \\
.04\end{array}$ & $\begin{array}{l}.175 \\
.179 \\
.169\end{array}$ & $\begin{array}{l}.528 \\
.562\end{array}$ & .8 & $\begin{array}{r}.982 \\
.990 \\
.987\end{array}$ & $\begin{array}{r}.999 \\
1.000 \\
.999\end{array}$ & & & & \\
\hline .30 & $\begin{array}{l}\text { MM } \\
\text { MU } \\
\text { MUT }\end{array}$ & & $\begin{array}{l}.18 \\
.17 \\
.17\end{array}$ & & & $\begin{array}{r}.9 \\
.9 \\
.9\end{array}$ & $\begin{array}{r}.999 \\
1.000 \\
1.000\end{array}$ & & & & \\
\hline $\begin{array}{l}.40 \\
.40\end{array}$ & $\begin{array}{l}\text { MM } \\
\text { MU } \\
\text { MUT }\end{array}$ & & $\begin{array}{l}.192 \\
.164 \\
.166\end{array}$ & & & & $\begin{array}{l}1.000 \\
1.000 \\
1.000\end{array}$ & & & & \\
\hline .50 & $\begin{array}{l}\text { MM } \\
\text { MU } \\
\text { MUT }\end{array}$ & & $\begin{array}{l}.21 \\
.15 \\
.17\end{array}$ & & & & $\begin{array}{l}1.000 \\
1.000 \\
1.000\end{array}$ & & & & \\
\hline .60 & $\begin{array}{l}\text { MM } \\
M U \\
\text { MUT }\end{array}$ & & $\begin{array}{l}.24 \\
.14 \\
.18\end{array}$ & & & $\begin{array}{l}9 \\
7 \\
7\end{array}$ & & & & & $\begin{array}{l}1.0 \\
1.0 \\
1.0\end{array}$ \\
\hline .70 & $\begin{array}{l}\text { MM } \\
\text { MU } \\
\text { MUT }\end{array}$ & $\begin{array}{l}.05 \\
.01 \\
.04\end{array}$ & $\begin{array}{l}.28 \\
.12 \\
.23\end{array}$ & & & $\begin{array}{r}1.0 \\
.9 \\
.9\end{array}$ & $\begin{array}{l}1.000 \\
1.000 \\
1.000\end{array}$ & & & & $\begin{array}{l}1.00 \\
1.00 \\
1.00\end{array}$ \\
\hline $\begin{array}{l}.80 \\
.80 \\
.80\end{array}$ & $\begin{array}{l}\text { MI } \\
\text { MU } \\
\text { MUT }\end{array}$ & $\begin{array}{l}.0 \\
.0 \\
.0\end{array}$ & $\begin{array}{l}.3 \\
.0 \\
.3\end{array}$ & $\begin{array}{l}.9 \\
.6 \\
.8\end{array}$ & $\begin{array}{l}.998 \\
.961 \\
.991\end{array}$ & $\begin{array}{r}1.000 \\
.999 \\
1.000\end{array}$ & $\begin{array}{l}1.000 \\
1.000 \\
1.000\end{array}$ & $\begin{array}{l}1 \\
1 \\
1\end{array}$ & $\begin{array}{l}1.000 \\
1.000 \\
1.000\end{array}$ & $\begin{array}{l}1.000 \\
1.000 \\
1.000\end{array}$ & $\begin{array}{l}1.0 \\
1.0 \\
1.0\end{array}$ \\
\hline .9 & $\begin{array}{l}\text { MM } \\
\text { MU } \\
\text { MUT }\end{array}$ & $\begin{array}{l}.0 \\
.0\end{array}$ & $\begin{array}{l}.630 \\
.063 \\
.625\end{array}$ & $\begin{array}{l}.9 \\
.6\end{array}$ & $\begin{array}{r}1.000 \\
.980 \\
1.000\end{array}$ & $\begin{array}{l}1.000 \\
1.000 \\
1.000\end{array}$ & $\begin{array}{l}1.000 \\
1.000 \\
1.000\end{array}$ & $\begin{array}{l}1.000 \\
1.000 \\
1.000\end{array}$ & $\begin{array}{l}1.000 \\
1.000 \\
1.000\end{array}$ & $\begin{array}{l}1.000 \\
1.000 \\
1.000\end{array}$ & 1. \\
\hline
\end{tabular}


POWER FOR 11 PAIRS

\begin{tabular}{|c|c|c|c|c|c|c|c|c|c|c|c|}
\hline \multicolumn{2}{|c|}{$\rho_{\mathrm{XY}}$ METHOD } & .0 & .25 & .50 & .75 & .00 & 1.25 & 1.50 & 1.75 & 2.00 & 2.25 \\
\hline $\begin{array}{l}.00 \\
.00 \\
.00 \\
.00\end{array}$ & $\begin{array}{l}\text { MM } \\
\text { MU } \\
\text { MUT } \\
\text { UU }\end{array}$ & $\begin{array}{l}.050 \\
.049 \\
.047 \\
.049\end{array}$ & $\begin{array}{l}.084 \\
.087 \\
.084 \\
.087\end{array}$ & $\begin{array}{l}.187 \\
.202 \\
.193 \\
.202\end{array}$ & $\begin{array}{l}.357 \\
.389 \\
.375 \\
.389\end{array}$ & $\begin{array}{l}.564 \\
.608 \\
.590 \\
.608\end{array}$ & $\begin{array}{l}.753 \\
.797 \\
.782 \\
.797\end{array}$ & $\begin{array}{l}.887 \\
.918 \\
.909 \\
.918\end{array}$ & $\begin{array}{r}.959 \\
.974 \\
.971 \\
.974\end{array}$ & $\begin{array}{r}.988 \\
.994 \\
.992 \\
.994\end{array}$ & $\begin{array}{r}.997 \\
.999 \\
.999 \\
.999\end{array}$ \\
\hline $\begin{array}{l}.10 \\
.10 \\
.10\end{array}$ & $\begin{array}{l}\text { MM } \\
\text { MU } \\
\text { MUT }\end{array}$ & 51 & & $\begin{array}{l}.188 \\
.200 \\
.192\end{array}$ & $\begin{array}{l}359 \\
389 \\
373\end{array}$ & & $\begin{array}{l}.756 \\
.796 \\
.782\end{array}$ & $\begin{array}{l}.888 \\
.917 \\
.908\end{array}$ & $\begin{array}{r}.959 \\
.974 \\
.970\end{array}$ & & $\begin{array}{r}.997 \\
.999 \\
.998\end{array}$ \\
\hline $\begin{array}{l}.20 \\
.20 \\
.20\end{array}$ & $\begin{array}{l}\text { MM } \\
\text { MU } \\
\text { MUT }\end{array}$ & $\begin{array}{l}050 \\
045\end{array}$ & $\begin{array}{l}.083 \\
.081\end{array}$ & $\begin{array}{l}.191 \\
.195\end{array}$ & $\begin{array}{l}.368 \\
385\end{array}$ & & $\begin{array}{l}.771 \\
.801 \\
.787\end{array}$ & $\begin{array}{l}.898 \\
.921 \\
.914\end{array}$ & $\begin{array}{r}.964 \\
.976 \\
.973\end{array}$ & & .9 \\
\hline $\begin{array}{l}.30 \\
.30 \\
.30\end{array}$ & $\begin{array}{l}\text { MM } \\
\text { MU } \\
\text { MUT }\end{array}$ & $\begin{array}{l}.051 \\
.041 \\
.044\end{array}$ & $\begin{array}{l}.08 \\
.07\end{array}$ & $\begin{array}{l}19 \\
19\end{array}$ & 384 & $\begin{array}{l}.603 \\
.612 \\
.600\end{array}$ & & $\begin{array}{l}.912 \\
.925 \\
.919\end{array}$ & $\begin{array}{l}.972 \\
.978 \\
.976\end{array}$ & & \\
\hline $\begin{array}{l}.40 \\
.40 \\
.40\end{array}$ & $\begin{array}{l}\text { MM } \\
\text { MU } \\
\text { MUT }\end{array}$ & .04 & .01 & .18 & .31 & $\begin{array}{l}.63 \\
.61 \\
.61\end{array}$ & $\begin{array}{l}.820 \\
.813 \\
.807\end{array}$ & .932 & $\begin{array}{l}.979 \\
.982\end{array}$ & & $\begin{array}{l}.95 \\
.95 \\
.95\end{array}$ \\
\hline $\begin{array}{l}.50 \\
.50 \\
.50\end{array}$ & $\begin{array}{l}\text { MM } \\
\text { MU } \\
\text { MUT }\end{array}$ & .05 & . & 23 & 年 & $\begin{array}{l}.685 \\
.622 \\
.634\end{array}$ & $\begin{array}{l}.860 \\
.825 \\
.829\end{array}$ & $\begin{array}{r}.940 \\
.941\end{array}$ & $\begin{array}{l}.989 \\
.985 \\
.985\end{array}$ & $\begin{array}{r}.998 \\
.998 \\
.997\end{array}$ & $\begin{array}{l}1.0 \\
1.0\end{array}$ \\
\hline $\begin{array}{l}.60 \\
.60 \\
.60\end{array}$ & $\begin{array}{l}\text { MM } \\
\text { MU } \\
\text { MUT }\end{array}$ & .03 & $\begin{array}{l}.103 \\
.047 \\
.079\end{array}$ & .210 & 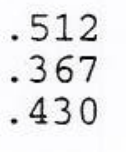 & .017 & .800 & .959 & $\begin{array}{l}.996 \\
.990 \\
.992\end{array}$ & $\begin{array}{r}1.000 \\
.999 \\
.999\end{array}$ & 1.00 \\
\hline $\begin{array}{l}.70 \\
.70 \\
.70\end{array}$ & $\begin{array}{l}\text { MM } \\
\text { MU } \\
\text { MUT }\end{array}$ & .04 & $\begin{array}{l}.112 \\
.032 \\
.093\end{array}$ & $\begin{array}{l}.316 \\
.133 \\
.258\end{array}$ & $\begin{array}{l}.60 \\
.35 \\
.51\end{array}$ & & & $\begin{array}{l}.992 \\
.965 \\
.980\end{array}$ & $\begin{array}{l}.999 \\
.994 \\
.997\end{array}$ & $\begin{array}{l}00 \\
99 \\
00\end{array}$ & 1.000 \\
\hline $\begin{array}{l}.80 \\
.80 \\
.80\end{array}$ & $\begin{array}{l}\text { MM } \\
\text { MU' } \\
\text { MUT }\end{array}$ & $\begin{array}{l}.05 \\
.00 \\
.05\end{array}$ & $\begin{array}{l}.145 \\
.019 \\
.137\end{array}$ & 42 & 68 & $\begin{array}{l}.940 \\
.662 \\
.890\end{array}$ & $\begin{array}{l}.992 \\
.892 \\
.975\end{array}$ & $\begin{array}{r}1.000 \\
.979 \\
.997\end{array}$ & $\begin{array}{r}.998 \\
1.000\end{array}$ & $\begin{array}{l}1.000 \\
1.000 \\
1.000\end{array}$ & $\begin{array}{l}1.00 \\
1.00 \\
1.00\end{array}$ \\
\hline $\begin{array}{l}.90 \\
.90 \\
.90\end{array}$ & $\begin{array}{l}\text { MM } \\
\text { MU } \\
\text { MUT }\end{array}$ & . & 39 & $\begin{array}{l}.071 \\
.682\end{array}$ & 1 & $\begin{array}{r}.998 \\
.694 \\
.994\end{array}$ & $\begin{array}{r}1.000 \\
.927 \\
1.000\end{array}$ & $\begin{array}{r}1.000 \\
.991 \\
1.000\end{array}$ & $\begin{array}{l}1.000 \\
1.000\end{array}$ & $\begin{array}{l}1.000 \\
1.000 \\
1.000\end{array}$ & $\begin{array}{l}1 \\
1\end{array}$ \\
\hline
\end{tabular}


POWER FOR 12 PAIRS

\begin{tabular}{|c|c|c|c|c|c|c|c|c|c|c|c|}
\hline \multicolumn{2}{|c|}{$\rho_{x y}$ METHOD } & .0 & .25 & .50 & .75 & 1.00 & 1.25 & 1.50 & 1.75 & 2.00 & 2.25 \\
\hline $\begin{array}{l}.00 \\
.00 \\
.00 \\
.00\end{array}$ & $\begin{array}{l}\text { MM } \\
\text { MU } \\
\text { MUT } \\
\text { UU }\end{array}$ & $\begin{array}{l}.050 \\
.050 \\
.047 \\
.050\end{array}$ & $\begin{array}{l}.087 \\
.091 \\
.085 \\
.091\end{array}$ & $\begin{array}{l}.202 \\
.215 \\
.202 \\
.215\end{array}$ & $\begin{array}{l}.388 \\
.419 \\
.399 \\
.419\end{array}$ & $\begin{array}{l}.605 \\
.649 \\
.626 \\
.649\end{array}$ & $\begin{array}{l}.795 \\
.832 \\
.815 \\
.832\end{array}$ & $\begin{array}{r}.916 \\
.939 \\
.931 \\
.939\end{array}$ & $\begin{array}{r}.973 \\
.983 \\
.980 \\
.983\end{array}$ & $\begin{array}{r}.994 \\
.996 \\
.996 \\
.996\end{array}$ & $\begin{array}{r}.999 \\
.999 \\
.999 \\
.999\end{array}$ \\
\hline $\begin{array}{l}.10 \\
10 \\
.10\end{array}$ & $\begin{array}{l}\text { MM } \\
\text { MU } \\
\text { MUT }\end{array}$ & $\begin{array}{l}.051 \\
.050 \\
.047\end{array}$ & $\begin{array}{l}.087 \\
.088 \\
.083\end{array}$ & $\begin{array}{l}.202 \\
.213 \\
.201\end{array}$ & $\begin{array}{l}.389 \\
.418 \\
.398\end{array}$ & $\begin{array}{l}.611 \\
.648 \\
.626\end{array}$ & $\begin{array}{l}.798 \\
.832 \\
.815\end{array}$ & $\begin{array}{r}.917 \\
.939 \\
.930\end{array}$ & $\begin{array}{l}.974 \\
.983 \\
.980\end{array}$ & $\begin{array}{l}.993 \\
.997 \\
.996\end{array}$ & $\begin{array}{r}.999 \\
1.000 \\
.999\end{array}$ \\
\hline $\begin{array}{l}.20 \\
.20 \\
.20\end{array}$ & $\begin{array}{l}\text { MM } \\
\text { MU } \\
\text { MUT }\end{array}$ & $\begin{array}{l}.049 \\
.045 \\
.044\end{array}$ & $\begin{array}{l}.088 \\
.085 \\
.081\end{array}$ & $\begin{array}{l}.207 \\
.211 \\
.201\end{array}$ & $\begin{array}{l}.401 \\
.415 \\
.398\end{array}$ & $\begin{array}{l}.625 \\
.651 \\
.630\end{array}$ & $\begin{array}{l}.814 \\
.841 \\
.825\end{array}$ & $\begin{array}{r}.928 \\
.945 \\
.937\end{array}$ & $\begin{array}{l}.978 \\
.985 \\
.982\end{array}$ & $\begin{array}{l}.995 \\
.997 \\
.996\end{array}$ & $\begin{array}{r}.999 \\
1.000 \\
.999\end{array}$ \\
\hline $\begin{array}{l}.30 \\
.30 \\
.30\end{array}$ & $\begin{array}{l}\text { MM } \\
\text { MU } \\
\text { MUT }\end{array}$ & $\begin{array}{l}.049 \\
.040 \\
.041\end{array}$ & $\begin{array}{l}.089 \\
.079 \\
.079\end{array}$ & $\begin{array}{l}.217 \\
.206 \\
.200\end{array}$ & $\begin{array}{l}.420 \\
.415 \\
.402\end{array}$ & $\begin{array}{l}.647 \\
.653 \\
.636\end{array}$ & $\begin{array}{l}.831 \\
.843 \\
.830\end{array}$ & $\begin{array}{r}.938 \\
.947 \\
.940\end{array}$ & $\begin{array}{l}.983 \\
.987 \\
.985\end{array}$ & $\begin{array}{l}.996 \\
.998 \\
.997\end{array}$ & $\begin{array}{r}.999 \\
1.000 \\
1.000\end{array}$ \\
\hline $\begin{array}{l}.40 \\
.40 \\
.40\end{array}$ & $\begin{array}{l}\text { MM } \\
\text { MU } \\
\text { MUT }\end{array}$ & $\begin{array}{l}.050 \\
.034 \\
.039\end{array}$ & $\begin{array}{l}.093 \\
.071 \\
.076\end{array}$ & $\begin{array}{l}.231 \\
.198 \\
.200\end{array}$ & $\begin{array}{l}.450 \\
.414 \\
.410\end{array}$ & $\begin{array}{l}.682 \\
.663 \\
.653\end{array}$ & $\begin{array}{l}.859 \\
.852 \\
.843\end{array}$ & $\begin{array}{r}.954 \\
.954 \\
.949\end{array}$ & $\begin{array}{l}.988 \\
.989 \\
.988\end{array}$ & $\begin{array}{l}.998 \\
.998 \\
.998\end{array}$ & $\begin{array}{l}1.00 \\
1.00 \\
1.00\end{array}$ \\
\hline .50 & $\begin{array}{l}\text { MM } \\
\text { MU } \\
\text { MUT }\end{array}$ & $\begin{array}{l}.050 \\
.027 \\
.038\end{array}$ & $\begin{array}{l}.099 \\
.062 \\
.076\end{array}$ & $\begin{array}{l}.25 \\
.18 \\
.20\end{array}$ & $\begin{array}{l}.489 \\
.409 \\
.425\end{array}$ & $\begin{array}{l}.729 \\
.667 \\
.674\end{array}$ & $\begin{array}{l}.896 \\
.862 \\
.863\end{array}$ & $\begin{array}{l}.971 \\
.961 \\
.959\end{array}$ & $\begin{array}{l}.994 \\
.992 \\
.992\end{array}$ & $\begin{array}{r}999 \\
.999 \\
.999\end{array}$ & $\begin{array}{l}1.0 \\
1.0 \\
1.0\end{array}$ \\
\hline $\begin{array}{l}.60 \\
.60 \\
.60\end{array}$ & $\begin{array}{l}\text { MM } \\
\text { MU } \\
\text { MUT }\end{array}$ & $\begin{array}{l}.050 \\
.018 \\
.037\end{array}$ & $\begin{array}{l}.109 \\
.049 \\
.083\end{array}$ & $\begin{array}{l}.289 \\
.172 \\
.228\end{array}$ & $\begin{array}{r}.405 \\
.466\end{array}$ & $\begin{array}{l}.796 \\
.681 \\
.721\end{array}$ & $\begin{array}{l}.935 \\
.879 \\
.896\end{array}$ & $\begin{array}{r}.986 \\
.969 \\
.973\end{array}$ & $\begin{array}{l}.998 \\
.995 \\
.995\end{array}$ & $\begin{array}{r}1.000 \\
.999 \\
.999\end{array}$ & $\begin{array}{l}1.000 \\
1.000 \\
1.000\end{array}$ \\
\hline $\begin{array}{l}.70 \\
.70 \\
.70\end{array}$ & $\begin{array}{l}\text { MM } \\
\text { MU } \\
\text { MUT }\end{array}$ & $\begin{array}{l}.049 \\
.009 \\
.041\end{array}$ & $\begin{array}{l}.120 \\
.034 \\
.099\end{array}$ & $\begin{array}{l}.343 \\
.148 \\
.281\end{array}$ & $\begin{array}{l}.647 \\
.397 \\
.554\end{array}$ & $\begin{array}{l}.877 \\
.694 \\
.801\end{array}$ & $\begin{array}{l}.974 \\
.901 \\
.942\end{array}$ & $\begin{array}{l}.997 \\
.980 \\
.989\end{array}$ & $\begin{array}{r}1.000 \\
.997 \\
.999\end{array}$ & $\begin{array}{l}1.000 \\
1.000 \\
1.000\end{array}$ & $\begin{array}{l}1.0 \\
1.0 \\
1.0\end{array}$ \\
\hline $\begin{array}{l}.80 \\
.80 \\
.80\end{array}$ & $\begin{array}{l}\text { MM } \\
\text { MU. } \\
\text { MUT }\end{array}$ & $\begin{array}{l}.050 \\
.003 \\
.049\end{array}$ & $\begin{array}{l}.154 \\
.020 \\
.144\end{array}$ & $\begin{array}{l}.460 \\
.123 \\
.418\end{array}$ & $\begin{array}{l}.793 \\
.389 \\
.731\end{array}$ & $\begin{array}{l}.959 \\
.719 \\
.920\end{array}$ & $\begin{array}{l}.996 \\
.926 \\
.986\end{array}$ & $\begin{array}{r}1.000 \\
.989 \\
.999\end{array}$ & $\begin{array}{r}1.000 \\
.999 \\
1.000\end{array}$ & $\begin{array}{l}1.000 \\
1.000 \\
1.000\end{array}$ & $\begin{array}{l}1.0 \\
1.0 \\
1.0\end{array}$ \\
\hline $\begin{array}{l}.9 \\
.9 \\
.9\end{array}$ & $\begin{array}{l}\text { MM } \\
\text { MU } \\
\text { MUT }\end{array}$ & $\begin{array}{l}.050 \\
.000 \\
.054\end{array}$ & .259 & $\begin{array}{l}.727 \\
.083 \\
.725\end{array}$ & $\begin{array}{l}373 \\
962\end{array}$ & $\begin{array}{l}.999 \\
.762 \\
.997\end{array}$ & $\begin{array}{r}1.000 \\
.957 \\
1.000\end{array}$ & $\begin{array}{r}.997 \\
1.000\end{array}$ & $\begin{array}{l}1.000 \\
1.000\end{array}$ & $\begin{array}{l}1.000 \\
1.000 \\
1.000\end{array}$ & \\
\hline
\end{tabular}


POWER FOR 13 PAIRS

\begin{tabular}{|c|c|c|c|c|c|c|c|c|c|c|c|}
\hline \multicolumn{2}{|c|}{$\rho_{x y}$ METHOD } & .0 & .25 & .50 & .75 & 1.00 & 1.25 & 1.50 & 1.75 & 2.00 & 25 \\
\hline $\begin{array}{l}.00 \\
.00 \\
.00 \\
.00\end{array}$ & $\begin{array}{l}\text { MM } \\
\text { MU } \\
\text { MUT } \\
\text { UU }\end{array}$ & $\begin{array}{l}.049 \\
.048 \\
.049 \\
.048\end{array}$ & $\begin{array}{l}.090 \\
.093 \\
.091 \\
.093\end{array}$ & $\begin{array}{l}.216 \\
.232 \\
.226 \\
.232\end{array}$ & $\begin{array}{l}.419 \\
.447 \\
.438 \\
.447\end{array}$ & $\begin{array}{l}.647 \\
.684 \\
.673 \\
.684\end{array}$ & $\begin{array}{l}.832 \\
.864 \\
.856 \\
.864\end{array}$ & $\begin{array}{l}.939 \\
.957 \\
.953 \\
.957\end{array}$ & $\begin{array}{l}.983 \\
.990 \\
.989 \\
.990\end{array}$ & $\begin{array}{l}.997 \\
.998 \\
.998 \\
.998\end{array}$ & $\begin{array}{l}.999 \\
1.000 \\
1.000 \\
1.000\end{array}$ \\
\hline $\begin{array}{l}.10 \\
.10 \\
.10\end{array}$ & $\begin{array}{l}\text { MM } \\
\text { MU } \\
\text { MUT }\end{array}$ & $\begin{array}{l}.050 \\
.049 \\
.049\end{array}$ & $\begin{array}{l}.091 \\
.093 \\
.092\end{array}$ & $\begin{array}{l}.21 \\
.23 \\
.22\end{array}$ & $\begin{array}{l}.424 \\
.450 \\
.440\end{array}$ & .651 & $\begin{array}{l}.836 \\
.864 \\
.856\end{array}$ & $\begin{array}{l}.940 \\
.956 \\
.952\end{array}$ & & & $\begin{array}{l}1.000 \\
1.000 \\
1.000\end{array}$ \\
\hline $\begin{array}{l}.20 \\
.20 \\
.20\end{array}$ & $\begin{array}{l}\text { MM } \\
\text { MU } \\
\text { MUT }\end{array}$ & $\begin{array}{l}.049 \\
.045 \\
.046\end{array}$ & $\begin{array}{l}.09 \\
.08 \\
.08\end{array}$ & $\begin{array}{l}.227 \\
.229 \\
.226\end{array}$ & $\begin{array}{l}.437 \\
.452 \\
.445\end{array}$ & .668 & $\begin{array}{l}.847 \\
.869 \\
.861\end{array}$ & $\begin{array}{l}.947 \\
.959 \\
.956\end{array}$ & & $\begin{array}{r}.998 \\
.999 \\
.999\end{array}$ & $\begin{array}{l}1.000 \\
1.000 \\
1.000\end{array}$ \\
\hline $\begin{array}{l}.30 \\
.30 \\
.30\end{array}$ & $\begin{array}{l}\text { MM } \\
\text { MU } \\
\text { MUT }\end{array}$ & $\begin{array}{l}.050 \\
.041 \\
.044\end{array}$ & $\begin{array}{l}.08 \\
.08 \\
.08\end{array}$ & .23 & $\begin{array}{l}.45 \\
.4 \\
.4\end{array}$ & .687 & $\begin{array}{l}.8 \\
.8 \\
.8\end{array}$ & .9 & & & $\begin{array}{l}1.000 \\
1.000 \\
1.000\end{array}$ \\
\hline $\begin{array}{l}.40 \\
.40 \\
.40\end{array}$ & $\begin{array}{l}\text { MM } \\
\text { MU } \\
\text { MUT }\end{array}$ & $\begin{array}{l}.04 \\
.03 \\
.04\end{array}$ & .09 & & $\begin{array}{l}.48 \\
.44 \\
.45\end{array}$ & .728 & .892 & & & $\begin{array}{r}.999 \\
.999 \\
.999\end{array}$ & $\begin{array}{l}1.000 \\
1.000 \\
1.000\end{array}$ \\
\hline $\begin{array}{l}.50 \\
.50 \\
.50\end{array}$ & $\begin{array}{l}\text { MM } \\
\text { MU } \\
\text { MUT }\end{array}$ & $\begin{array}{l}.050 \\
.025 \\
.039\end{array}$ & .1 & & $\begin{array}{l}.52 \\
.44 \\
.47\end{array}$ & .770 & & & & $\begin{array}{l}1.0 \\
1.0 \\
1.0\end{array}$ & $\begin{array}{l}1.000 \\
1.000 \\
1.000\end{array}$ \\
\hline $\begin{array}{l}.60 \\
.60 \\
.60\end{array}$ & $\begin{array}{l}\text { MM } \\
\text { MU } \\
\text { MUT }\end{array}$ & $\begin{array}{l}.04 \\
.01 \\
.03\end{array}$ & .1 & & & .8 & & .9 & $\begin{array}{r}9 \\
.9 \\
.9\end{array}$ & $\begin{array}{l}1.000 \\
1.000 \\
1.000\end{array}$ & $\begin{array}{l}1.000 \\
1.000 \\
1.000\end{array}$ \\
\hline $\begin{array}{l}.70 \\
.70 \\
.70\end{array}$ & $\begin{array}{l}\text { MM } \\
\text { MU } \\
\text { MUT }\end{array}$ & $\begin{array}{l}.051 \\
.010 \\
.046\end{array}$ & $\begin{array}{l}.13 \\
.03 \\
.11\end{array}$ & & $\begin{array}{l}.65 \\
.43 \\
.63\end{array}$ & $\begin{array}{r}.906 \\
.743 \\
.849\end{array}$ & $\begin{array}{l}3 \\
7 \\
3\end{array}$ & $\begin{array}{r}.9 \\
.9 \\
.9\end{array}$ & $\begin{array}{r}.0 \\
.9 \\
.9\end{array}$ & $\begin{array}{l}1.000 \\
1.000 \\
1.000\end{array}$ & $\begin{array}{l}1.000 \\
1.000 \\
1.000\end{array}$ \\
\hline $\begin{array}{l}.80 \\
.80 \\
.80\end{array}$ & $\begin{array}{l}\text { MM } \\
\text { MU. } \\
\text { MUT }\end{array}$ & $\begin{array}{l}.051 \\
.003 \\
.051\end{array}$ & $\begin{array}{l}.16 \\
.02 \\
.16\end{array}$ & $\begin{array}{l}.45 \\
.13 \\
.46\end{array}$ & $\begin{array}{l}.83 \\
.43 \\
.78\end{array}$ & $\begin{array}{r}.973 \\
.772 \\
.950\end{array}$ & $\begin{array}{l}8 \\
9 \\
3\end{array}$ & $\begin{array}{r}1.0 \\
.9 \\
1.0\end{array}$ & $\begin{array}{l}1.000 \\
1.000 \\
1.000\end{array}$ & $\begin{array}{l}1.000 \\
1.000 \\
1.000\end{array}$ & $\begin{array}{l}1.000 \\
1.000 \\
1.000\end{array}$ \\
\hline $\begin{array}{l}.90 \\
.90 \\
.90\end{array}$ & $\begin{array}{l}\text { MM } \\
\text { MU } \\
\text { MUT }\end{array}$ & $\begin{array}{l}.049 \\
.000 \\
.054\end{array}$ & $\begin{array}{l}.270 \\
.006 \\
.284\end{array}$ & $\begin{array}{l}.765 \\
.094 \\
.772\end{array}$ & $\begin{array}{r}.980 \\
.426 \\
.977\end{array}$ & $\begin{array}{r}.999 \\
.817 \\
.999\end{array}$ & $\begin{array}{r}1.000 \\
.976 \\
1.000\end{array}$ & $\begin{array}{r}1.000 \\
.999 \\
1.000\end{array}$ & $\begin{array}{l}1.000 \\
1.000 \\
1.000\end{array}$ & $\begin{array}{l}1.000 \\
1.000 \\
1.000\end{array}$ & $\begin{array}{l}1.000 \\
1.000 \\
1.000\end{array}$ \\
\hline
\end{tabular}


POWER FOR 14 PAIRS

\begin{tabular}{|c|c|c|c|c|c|c|c|c|c|c|c|}
\hline \multicolumn{2}{|c|}{$\rho_{x y}$ METHOD } & \multicolumn{2}{|c|}{$-10-10-1$} & .50 & \multicolumn{3}{|c|}{$\begin{array}{l}.75-\text { EFFECT SIZE } \\
.751 .00 \quad 1.25\end{array}$} & 1.50 & 1.75 & 2.00 & 2.25 \\
\hline $\begin{array}{l}.00 \\
.00 \\
.00 \\
.00\end{array}$ & $\begin{array}{l}\text { MM } \\
\text { MU } \\
\text { MUT } \\
\text { UU }\end{array}$ & $\begin{array}{l}.049 \\
.049 \\
.048 \\
.049\end{array}$ & $\begin{array}{l}.093 \\
.096 \\
.094 \\
.096\end{array}$ & $\begin{array}{l}.231 \\
.246 \\
.240 \\
.246\end{array}$ & $\begin{array}{l}.451 \\
.480 \\
.471 \\
.480\end{array}$ & $\begin{array}{l}.687 \\
.720 \\
.711 \\
.720\end{array}$ & $\begin{array}{l}.862 \\
.889 \\
.883 \\
.889\end{array}$ & $\begin{array}{l}.955 \\
.969 \\
.966 \\
.969\end{array}$ & $\begin{array}{r}.989 \\
.994 \\
.993 \\
.994\end{array}$ & $\begin{array}{r}.998 \\
.999 \\
.999 \\
.999\end{array}$ & $\begin{array}{l}1.000 \\
1.000 \\
1.000 \\
1.000\end{array}$ \\
\hline $\begin{array}{l}.10 \\
.10 \\
.10\end{array}$ & $\begin{array}{l}\text { MM } \\
\text { MU } \\
\text { MUT }\end{array}$ & $\begin{array}{l}.050 \\
.050 \\
.049\end{array}$ & $\begin{array}{l}.094 \\
.097 \\
.095\end{array}$ & $\begin{array}{l}.234 \\
.246 \\
.241\end{array}$ & $\begin{array}{l}.456 \\
.482 \\
.473\end{array}$ & $\begin{array}{l}.693 \\
.723 \\
.714\end{array}$ & $\begin{array}{l}.867 \\
.892 \\
.886\end{array}$ & $\begin{array}{r}.957 \\
.969 \\
.967\end{array}$ & $\begin{array}{l}.990 \\
.994 \\
.993\end{array}$ & $\begin{array}{r}.998 \\
.999 \\
.999\end{array}$ & $\begin{array}{l}1.000 \\
1.000 \\
1.000\end{array}$ \\
\hline $\begin{array}{l}.20 \\
.20 \\
.20\end{array}$ & $\begin{array}{l}\text { MM } \\
\text { MU } \\
\text { MUT }\end{array}$ & $\begin{array}{l}.049 \\
.046 \\
.046\end{array}$ & $\begin{array}{l}.096 \\
.094 \\
.094\end{array}$ & $\begin{array}{l}.238 \\
.242 \\
.239\end{array}$ & $\begin{array}{l}.464 \\
.479 \\
.472\end{array}$ & $\begin{array}{l}.703 \\
.725 \\
.717\end{array}$ & $\begin{array}{l}.877 \\
.893 \\
.888\end{array}$ & $\begin{array}{l}.961 \\
.970 \\
.968\end{array}$ & $\begin{array}{r}.991 \\
.994 \\
.994\end{array}$ & $\begin{array}{r}.999 \\
.999 \\
.999\end{array}$ & $\begin{array}{l}1.000 \\
1.000 \\
1.000\end{array}$ \\
\hline $\begin{array}{l}.30 \\
.30 \\
.30\end{array}$ & $\begin{array}{l}\text { MM } \\
\text { MU } \\
\text { MUT }\end{array}$ & $\begin{array}{l}.050 \\
.040 \\
.043\end{array}$ & $\begin{array}{l}.099 \\
.086 \\
.089\end{array}$ & $\begin{array}{l}.251 \\
.238 \\
.238\end{array}$ & $\begin{array}{l}.488 \\
.482 \\
.479\end{array}$ & $\begin{array}{l}.728 \\
.731 \\
.727\end{array}$ & $\begin{array}{l}.892 \\
.898 \\
.895\end{array}$ & $\begin{array}{r}.969 \\
.974 \\
.972\end{array}$ & $\begin{array}{l}.994 \\
.995 \\
.995\end{array}$ & $\begin{array}{r}.999 \\
.999 \\
.999\end{array}$ & $\begin{array}{l}1.000 \\
1.000 \\
1.000\end{array}$ \\
\hline $\begin{array}{l}.40 \\
.40 \\
.40\end{array}$ & $\begin{array}{l}\text { MM } \\
\text { MU } \\
\text { MUT }\end{array}$ & $\begin{array}{l}.050 \\
.034 \\
.040\end{array}$ & $\begin{array}{l}.102 \\
.078 \\
.086\end{array}$ & $\begin{array}{l}.267 \\
.231 \\
.240\end{array}$ & $\begin{array}{l}.519 \\
.480 \\
.486\end{array}$ & $\begin{array}{l}.762 \\
.737 \\
.740\end{array}$ & $\begin{array}{l}.915 \\
.908 \\
.908\end{array}$ & $\begin{array}{r}.980 \\
.979 \\
.979\end{array}$ & $\begin{array}{r}.997 \\
.997 \\
.996\end{array}$ & $\begin{array}{l}1.000 \\
1.000 \\
1.000\end{array}$ & $\begin{array}{l}1.000 \\
1.000 \\
1.000\end{array}$ \\
\hline $\begin{array}{l}.50 \\
.50 \\
.50\end{array}$ & $\begin{array}{l}\text { MM } \\
\text { MU } \\
\text { MUT }\end{array}$ & $\begin{array}{l}.050 \\
.025 \\
.038\end{array}$ & $\begin{array}{l}.110 \\
.068 \\
.087\end{array}$ & $\begin{array}{l}.295 \\
.219 \\
.248\end{array}$ & $\begin{array}{l}.565 \\
.480 \\
.508\end{array}$ & $\begin{array}{l}.804 \\
.748 \\
.763\end{array}$ & $\begin{array}{l}.940 \\
.917 \\
.922\end{array}$ & $\begin{array}{l}.988 \\
.982 \\
.983\end{array}$ & $\begin{array}{r}.999 \\
.998 \\
.998\end{array}$ & $\begin{array}{l}1.000 \\
1.000 \\
1.000\end{array}$ & $\begin{array}{l}1.000 \\
1.000 \\
1.000\end{array}$ \\
\hline $\begin{array}{l}.60 \\
.60 \\
.60\end{array}$ & $\begin{array}{l}\text { MM } \\
\text { MU } \\
\text { MUT }\end{array}$ & $\begin{array}{r}.050 \\
.017 \\
.039\end{array}$ & $\begin{array}{l}.119 \\
.055 \\
.093\end{array}$ & $\begin{array}{l}.332 \\
.201 \\
.271\end{array}$ & $\begin{array}{l}.632 \\
.477 \\
.551\end{array}$ & $\begin{array}{l}.864 \\
.763 \\
.808\end{array}$ & $\begin{array}{r}.969 \\
.933 \\
.947\end{array}$ & $\begin{array}{l}.995 \\
.989 \\
.991\end{array}$ & $\begin{array}{r}1.000 \\
.999 \\
.999\end{array}$ & $\begin{array}{l}1.000 \\
1.000 \\
1.000\end{array}$ & $\begin{array}{l}1.000 \\
1.000 \\
1.000\end{array}$ \\
\hline $\begin{array}{l}.70 \\
.70 \\
.70\end{array}$ & $\begin{array}{l}\text { MM } \\
\text { MU } \\
\text { MUT }\end{array}$ & $\begin{array}{l}.050 \\
.008 \\
.043\end{array}$ & $\begin{array}{l}.138 \\
.040 \\
.117\end{array}$ & $\begin{array}{l}.404 \\
.182 \\
.344\end{array}$ & $\begin{array}{l}.729 \\
.476 \\
.649\end{array}$ & $\begin{array}{l}.928 \\
.785 \\
.878\end{array}$ & $\begin{array}{r}.989 \\
.948 \\
.975\end{array}$ & $\begin{array}{l}.999 \\
.994 \\
.997\end{array}$ & $\begin{array}{l}1.000 \\
1.000 \\
1.000\end{array}$ & $\begin{array}{l}1.000 \\
1.000 \\
1.000\end{array}$ & $\begin{array}{l}1.000 \\
1.000 \\
1.000\end{array}$ \\
\hline $\begin{array}{l}.80 \\
.80 \\
.80\end{array}$ & $\begin{array}{l}\text { MM } \\
\text { MU } \\
\text { MUT }\end{array}$ & $\begin{array}{l}.049 \\
.003 \\
.051\end{array}$ & $\begin{array}{l}.176 \\
.022 \\
.171\end{array}$ & $\begin{array}{l}.533 \\
.152 \\
.502\end{array}$ & $\begin{array}{l}.863 \\
.477 \\
.821\end{array}$ & $\begin{array}{l}.982 \\
.816 \\
.963\end{array}$ & $\begin{array}{r}.999 \\
.969 \\
.996\end{array}$ & $\begin{array}{r}1.000 \\
.997 \\
1.000\end{array}$ & $\begin{array}{l}1.000 \\
1.000 \\
1.000\end{array}$ & $\begin{array}{l}1.000 \\
1.000 \\
1.000\end{array}$ & $\begin{array}{l}1.000 \\
1.000 \\
1.000\end{array}$ \\
\hline $\begin{array}{l}.90 \\
.90 \\
.90\end{array}$ & $\begin{array}{l}\text { MM } \\
\text { MU } \\
\text { MUT }\end{array}$ & $\begin{array}{l}.049 \\
.000 \\
.055\end{array}$ & $\begin{array}{l}.290 \\
.007 \\
.306\end{array}$ & $\begin{array}{l}.800 \\
.106 \\
.807\end{array}$ & $\begin{array}{l}.988 \\
.475 \\
.986\end{array}$ & $\begin{array}{r}1.000 \\
.864 \\
1.000\end{array}$ & $\begin{array}{r}1.000 \\
.987 \\
1.000\end{array}$ & $\begin{array}{l}1.000 \\
1.000 \\
1.000\end{array}$ & $\begin{array}{l}1.000 \\
1.000 \\
1.000\end{array}$ & $\begin{array}{l}1.000 \\
1.000 \\
1.000\end{array}$ & $\begin{array}{l}1.000 \\
1.000 \\
1.000\end{array}$ \\
\hline
\end{tabular}


POWER FOR 15 PAIRS

\begin{tabular}{|c|c|c|c|c|c|c|c|c|c|c|c|}
\hline $\mathrm{ky}_{\mathrm{K}} \mathrm{MB}$ & THOD & .0 & .25 & .50 & .75 & 1.0 & 1.25 & 1.50 & 1.75 & 2.00 & 25 \\
\hline $\begin{array}{l}.00 \\
.00 \\
.00 \\
.00\end{array}$ & $\begin{array}{l}\text { MM } \\
\text { MU } \\
\text { MUT } \\
\text { UU }\end{array}$ & $\begin{array}{l}.050 \\
.050 \\
.048 \\
.050\end{array}$ & $\begin{array}{l}.098 \\
.102 \\
.098 \\
.102\end{array}$ & $\begin{array}{l}.248 \\
.263 \\
.254 \\
.263\end{array}$ & $\begin{array}{l}.480 \\
.508 \\
.496 \\
.508\end{array}$ & $\begin{array}{l}.720 \\
.752 \\
.740 \\
.752\end{array}$ & $\begin{array}{l}.888 \\
.910 \\
.903 \\
.910\end{array}$ & $\begin{array}{l}.968 \\
.977 \\
.974 \\
.977\end{array}$ & & $\begin{array}{l}.999 \\
.999 \\
.999 \\
.999\end{array}$ & $\begin{array}{l}1.00 \\
1.00 \\
1.00 \\
1.00\end{array}$ \\
\hline $\begin{array}{l}10 \\
.10 \\
.10\end{array}$ & $\begin{array}{l}\text { MM } \\
\text { MU } \\
\text { MUT }\end{array}$ & $\begin{array}{l}.049 \\
.049 \\
.047\end{array}$ & $\begin{array}{l}.098 \\
.100 \\
.097\end{array}$ & $\begin{array}{l}.250 \\
.261 \\
.252\end{array}$ & $\begin{array}{r}.484 \\
.510 \\
.497\end{array}$ & .727 & $\begin{array}{r}.892 \\
.912 \\
.905\end{array}$ & $\begin{array}{l}.969 \\
.978 \\
.976\end{array}$ & & $\begin{array}{r}.999 \\
1.000 \\
1.000\end{array}$ & $\begin{array}{l}1.00 \\
1.00\end{array}$ \\
\hline $\begin{array}{l}.20 \\
.20 \\
.20\end{array}$ & $\begin{array}{l}\text { MM } \\
\text { MU } \\
\text { MUT }\end{array}$ & $\begin{array}{l}.04 \\
.04 \\
.04\end{array}$ & $\begin{array}{l}.099 \\
.097 \\
.094\end{array}$ & $\begin{array}{l}.256 \\
.257 \\
.252\end{array}$ & .497 & .739 & .902 & $\begin{array}{r}.973 \\
.979 \\
.977\end{array}$ & & $\begin{array}{r}.999 \\
1.000 \\
1.000\end{array}$ & $\begin{array}{l}1.000 \\
1.000 \\
1.000\end{array}$ \\
\hline $\begin{array}{l}.30 \\
.30 \\
.30\end{array}$ & $\begin{array}{l}\text { MM } \\
\text { MU } \\
\text { MUT }\end{array}$ & $\begin{array}{l}.04 \\
.04 \\
.04\end{array}$ & $\begin{array}{l}.10 \\
.09 \\
.09\end{array}$ & $\begin{array}{l}.26 \\
.25\end{array}$ & 5 & .764 & & $\begin{array}{l}.979 \\
.982 \\
.980\end{array}$ & & $\begin{array}{l}1.000 \\
1.000 \\
1.000\end{array}$ & $\begin{array}{l}1.000 \\
1.000 \\
1.000\end{array}$ \\
\hline $\begin{array}{l}.40 \\
.40 \\
.40\end{array}$ & $\begin{array}{l}\text { MM } \\
\text { MU } \\
\text { MUT }\end{array}$ & $\begin{array}{l}.05 \\
.03 \\
.04\end{array}$ & $\begin{array}{l}.10 \\
.08 \\
.09\end{array}$ & 2 & & .795 & .9 & $\begin{array}{l}.986 \\
.985 \\
.985\end{array}$ & & $\begin{array}{l}1.000 \\
1.000 \\
1.000\end{array}$ & $\begin{array}{l}1.000 \\
1.000 \\
1.000\end{array}$ \\
\hline $\begin{array}{l}.50 \\
.50 \\
.50\end{array}$ & $\begin{array}{l}\text { MM } \\
\text { MU } \\
\text { MUT }\end{array}$ & $\begin{array}{l}.05 \\
.02 \\
.03\end{array}$ & $\begin{array}{l}.11 \\
.07 \\
.09\end{array}$ & 3 & & .835 & .9 & $\begin{array}{l}.992 \\
.988 \\
.989\end{array}$ & & $\begin{array}{l}1.000 \\
1.000 \\
1.000\end{array}$ & $\begin{array}{l}1.000 \\
1.000 \\
1.000\end{array}$ \\
\hline $\begin{array}{l}.60 \\
.60 \\
.60\end{array}$ & $\begin{array}{l}\text { MM } \\
\text { MU } \\
\text { MUT }\end{array}$ & $\begin{array}{l}.05 \\
.01 \\
.04\end{array}$ & $\begin{array}{l}.12 \\
.05 \\
.10\end{array}$ & 3 & 6 & .889 & .9 & $\begin{array}{r}.998 \\
.993 \\
.995\end{array}$ & $\begin{array}{r}1.0 \\
.9 \\
1.0\end{array}$ & $\begin{array}{l}1.000 \\
1.000 \\
1.000\end{array}$ & $\begin{array}{l}1.000 \\
1.000 \\
1.000\end{array}$ \\
\hline $\begin{array}{l}.70 \\
.70 \\
.70\end{array}$ & $\begin{array}{l}\text { MM } \\
\text { MU } \\
\text { MUT }\end{array}$ & $\begin{array}{l}.05 \\
.00 \\
.04\end{array}$ & $\begin{array}{l}.14 \\
.04 \\
.12\end{array}$ & $\begin{array}{l}42 \\
19 \\
37\end{array}$ & & .94 & $\begin{array}{r}.9 \\
.9 \\
.9\end{array}$ & $\begin{array}{r}1.000 \\
.996 \\
.998\end{array}$ & $\begin{array}{l}1.000 \\
1.000 \\
1.000\end{array}$ & $\begin{array}{l}1.000 \\
1.000 \\
1.000\end{array}$ & $\begin{array}{l}1.000 \\
1.000 \\
1.000\end{array}$ \\
\hline $\begin{array}{l}.80 \\
.80 \\
.80\end{array}$ & $\begin{array}{l}\text { MM } \\
\text { MU } \\
\text { MUT }\end{array}$ & $\begin{array}{l}.04 \\
.00 \\
.05\end{array}$ & $\begin{array}{l}.18 \\
.02 \\
.18\end{array}$ & $\begin{array}{l}.56 \\
.16 \\
.54\end{array}$ & $\begin{array}{l}.88 \\
.52 \\
.85\end{array}$ & $\begin{array}{r}.989 \\
.853 \\
.977\end{array}$ & $\begin{array}{l}.0 \\
.9 \\
.9\end{array}$ & $\begin{array}{r}1.000 \\
.999 \\
1.000\end{array}$ & $\begin{array}{l}1.000 \\
1.000 \\
1.000\end{array}$ & $\begin{array}{l}1.000 \\
1.000 \\
1.000\end{array}$ & $\begin{array}{l}1.000 \\
1.000 \\
1.000\end{array}$ \\
\hline $\begin{array}{l}.90 \\
.90 \\
.90\end{array}$ & $\begin{array}{l}\text { MM } \\
M U \\
\text { MUT }\end{array}$ & $\begin{array}{l}.04 \\
.00 \\
.05\end{array}$ & $\begin{array}{l}.308 \\
.007 \\
.321\end{array}$ & .83 & $\begin{array}{l}.992 \\
.527 \\
.991\end{array}$ & $\begin{array}{r}1.000 \\
.900 \\
1.000\end{array}$ & $\begin{array}{r}1.000 \\
.993 \\
1.000\end{array}$ & $\begin{array}{l}1.000 \\
1.000 \\
1.000\end{array}$ & $\begin{array}{l}1.000 \\
1.000 \\
1.000\end{array}$ & $\begin{array}{l}1.000 \\
1.000 \\
1.000\end{array}$ & $\begin{array}{l}1.000 \\
1.000 \\
1.000\end{array}$ \\
\hline
\end{tabular}


POWER FOR 16 PAIRS

PIDTHOD

$\begin{array}{llllllllllll}.00 & \text { MM } & .049 & .102 & .261 & .510 & .752 & .909 & .977 & .996 & .999 & 1.000\end{array}$

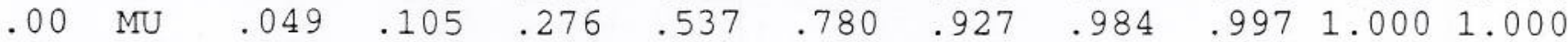

$\begin{array}{llllllllllll}.00 & \text { MUT } & .045 & .097 & .260 & .516 & .763 & .918 & .981 & .997 & 1.000 & 1.000\end{array}$

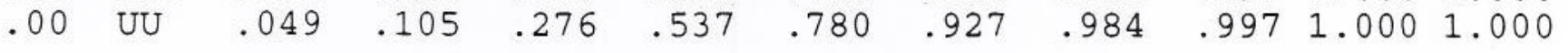





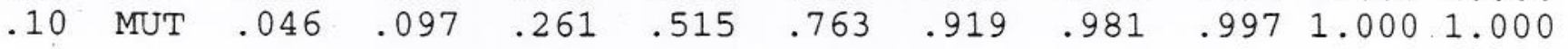

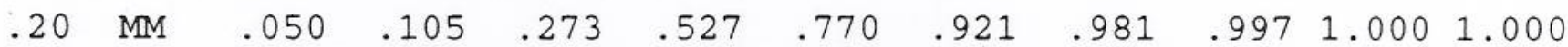

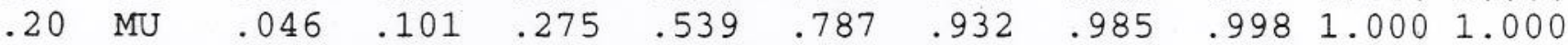



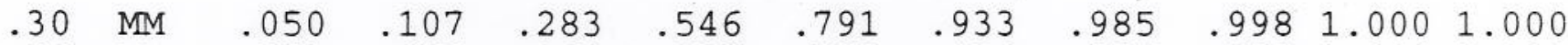

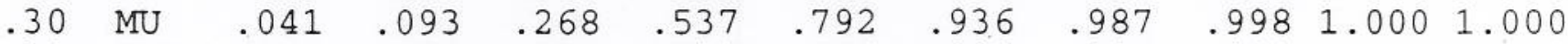



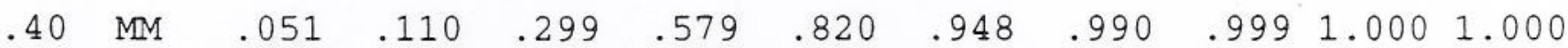

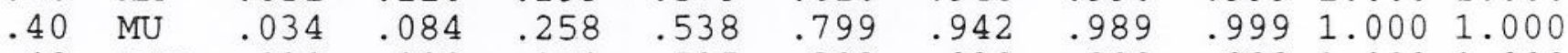



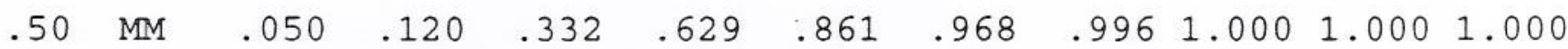

$\begin{array}{llllllllllll}.50 & \text { MU } & .025 & .075 & .251 & .544 & .813 & .952 & .993 & .999 & 1.000 & 1.000\end{array}$

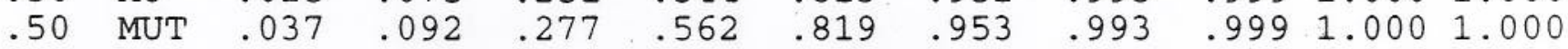

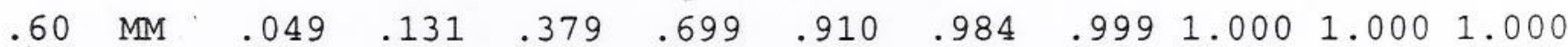

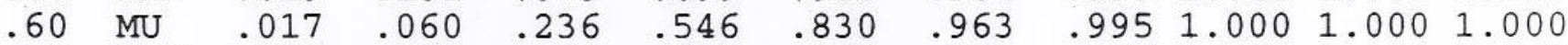

$\begin{array}{llllllllllll}.60 & \text { MUT } & .037 & .102 & .310 & .616 & .863 & .971 & .997 & 1.000 & 1.000 & 1.000\end{array}$

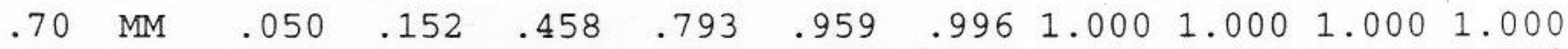

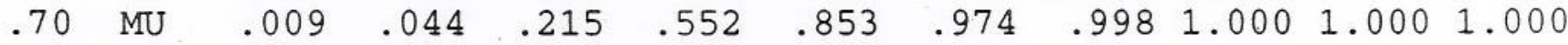

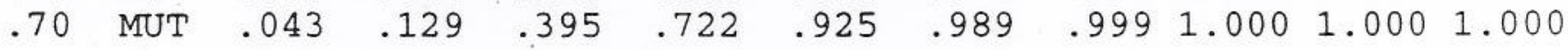

$\begin{array}{llllllllllll}.80 & \text { MM } & .050 & .196 & .596 & .910 & .993 & 1.000 & 1.000 & 1.000 & 1.000 & 1.000\end{array}$

$\begin{array}{llllllllllll}.80 & \text { MU } & .002 & .025 & .184 & .559 & .884 & .987 & .999 & 1.000 & 1.000 & 1.000\end{array}$

$\begin{array}{llllllllllll}.80 & \text { MUT } & .049 & .187 & .565 & .881 & .985 & .999 & 1.000 & 1.000 & 1.000 & 1.000\end{array}$

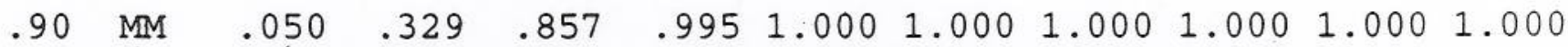

.90 MU $\quad \begin{array}{lllllllllll}.000 & .007 & .139 & .578 & .929 & .996 & 1.000 & 1.000 & 1.000 & 1.000\end{array}$




POWER FOR 17 PAIRS

\begin{tabular}{|c|c|c|c|c|c|c|c|c|c|c|c|}
\hline$\rho_{X Y} M$ & HOD & .0 & .25 & .50 & .75 & 1.00 & 1.25 & 1.50 & 1.75 & 2.00 & 2.25 \\
\hline $\begin{array}{l}.00 \\
.00 \\
.00 \\
.00\end{array}$ & $\begin{array}{l}\text { MM } \\
\text { MU } \\
\text { MUT } \\
\text { UU }\end{array}$ & $\begin{array}{l}.049 \\
.050 \\
.047 \\
.050\end{array}$ & $\begin{array}{l}.106 \\
.110 \\
.105 \\
.110\end{array}$ & $\begin{array}{l}.278 \\
.295 \\
.284 \\
.295\end{array}$ & $\begin{array}{l}.539 \\
.567 \\
.553 \\
.567\end{array}$ & $\begin{array}{l}.782 \\
.810 \\
.798 \\
.810\end{array}$ & $\begin{array}{l}.928 \\
.944 \\
.938 \\
.944\end{array}$ & & $\begin{array}{l}.997 \\
.998 \\
.998 \\
.998\end{array}$ & & $\begin{array}{l}1.000 \\
1.000 \\
1.000 \\
1.000\end{array}$ \\
\hline $\begin{array}{l}10 \\
.10 \\
.10\end{array}$ & $\begin{array}{l}\text { MM } \\
\text { MU } \\
\text { MUT }\end{array}$ & $\begin{array}{l}.049 \\
.049 \\
.047\end{array}$ & $\begin{array}{l}.105 \\
.110 \\
.105\end{array}$ & $\begin{array}{l}.282 \\
.296 \\
.285\end{array}$ & $\begin{array}{l}.543 \\
.569 \\
.554\end{array}$ & $\begin{array}{l}.786 \\
.811 \\
.799\end{array}$ & .930 & & $\begin{array}{l}.998 \\
.999 \\
.998\end{array}$ & & $\begin{array}{l}1.000 \\
1.000 \\
1.000\end{array}$ \\
\hline $\begin{array}{l}.20 \\
.20 \\
.20\end{array}$ & $\begin{array}{l}\text { MM } \\
\text { MU } \\
\text { MUT }\end{array}$ & $\begin{array}{l}.050 \\
.047 \\
.046\end{array}$ & $\begin{array}{l}.108 \\
.105 \\
.102\end{array}$ & $\begin{array}{l}.28 \\
.28 \\
.28\end{array}$ & .553 & $\begin{array}{r}.799 \\
.814 \\
.805\end{array}$ & $\begin{array}{r}.937 \\
.947 \\
.942\end{array}$ & $\begin{array}{r}.987 \\
.990 \\
.989 .\end{array}$ & $\begin{array}{r}.998 \\
.999 \\
.999\end{array}$ & & $\begin{array}{l}1 . \\
1 . \\
1 .\end{array}$ \\
\hline $\begin{array}{l}.30 \\
.30 \\
.30\end{array}$ & $\begin{array}{l}\text { MM } \\
\text { MU } \\
\text { MUT }\end{array}$ & $\begin{array}{l}.050 \\
.041 \\
.042\end{array}$ & $\begin{array}{l}.11 \\
.09 \\
.10\end{array}$ & $\begin{array}{l}.38 \\
.28 \\
.28\end{array}$ & .57 & .81 & .9 & $\begin{array}{r}.990 \\
.992 \\
.991\end{array}$ & $\begin{array}{l}.999 \\
.999 \\
.999\end{array}$ & & $\begin{array}{l}1 . \\
1 . \\
1 .\end{array}$ \\
\hline $\begin{array}{l}.40 \\
.40 \\
.40\end{array}$ & $\begin{array}{l}\text { MM } \\
\text { MU } \\
\text { MUT }\end{array}$ & $\begin{array}{l}.051 \\
.034 \\
.040\end{array}$ & $\begin{array}{l}.11 \\
.09 \\
.09\end{array}$ & .3 & 6 & .848 & .962 & $\begin{array}{r}.9 \\
.9 \\
.9\end{array}$ & $\begin{array}{l}.999 \\
.999 \\
.999\end{array}$ & & $\begin{array}{l}1.000 \\
1.000 \\
1.000\end{array}$ \\
\hline $\begin{array}{l}.50 \\
.50 \\
.50\end{array}$ & $\begin{array}{l}\text { MM } \\
\text { MU } \\
\text { MUT }\end{array}$ & $\begin{array}{l}.049 \\
.026 \\
.038\end{array}$ & $\begin{array}{l}.12 \\
.07 \\
.09\end{array}$ & .2 & 6 & $\begin{array}{l}.841 \\
.851\end{array}$ & 0 & $\begin{array}{l}.997 \\
.995 \\
.995\end{array}$ & $\begin{array}{l}1.000 \\
1.000 \\
1.000\end{array}$ & & $\begin{array}{l}1.000 \\
1.000\end{array}$ \\
\hline $\begin{array}{l}.60 \\
.60 \\
.60\end{array}$ & $\begin{array}{l}\text { MM } \\
\text { MU } \\
\text { MUT }\end{array}$ & $\begin{array}{l}.050 \\
.017 \\
.040\end{array}$ & $\begin{array}{l}.13 \\
.06 \\
.11\end{array}$ & .3 & .7 & $\begin{array}{l}.92 \\
.85 \\
.89\end{array}$ & .980 & $\begin{array}{l}.999 \\
.997 \\
.998\end{array}$ & $\begin{array}{l}1.000 \\
1.000 \\
1.000\end{array}$ & & $\begin{array}{l}1.000 \\
1.000\end{array}$ \\
\hline $\begin{array}{l}.70 \\
.70 \\
.70\end{array}$ & $\begin{array}{l}\text { MM } \\
\text { MU } \\
\text { MUT }\end{array}$ & $\begin{array}{l}.00 \\
.04\end{array}$ & $\begin{array}{l}.15 \\
.04 \\
.13\end{array}$ & .4 & & $\begin{array}{l}.969 \\
.879 \\
.944\end{array}$ & $\begin{array}{l}.998 \\
.983 \\
.993\end{array}$ & $\begin{array}{r}1.000 \\
.999 \\
1.000\end{array}$ & $\begin{array}{l}1.000 \\
1.000 \\
1.000\end{array}$ & & $\begin{array}{l}1.000 \\
1.000 \\
1.000\end{array}$ \\
\hline $\begin{array}{l}.80 \\
.80 \\
.80\end{array}$ & $\begin{array}{l}\text { MM } \\
\text { MU } \\
\text { MUT }\end{array}$ & $\begin{array}{l}.051 \\
.003 \\
.052\end{array}$ & $\begin{array}{l}.2 \\
.02 \\
.2\end{array}$ & $\begin{array}{l}.6 \\
.2 \\
.6\end{array}$ & .6 & $\begin{array}{r}.995 \\
.911 \\
.991\end{array}$ & $\begin{array}{r}1.000 \\
.992 \\
1.000\end{array}$ & $\begin{array}{l}1.000 \\
1.000 \\
1.000\end{array}$ & $\begin{array}{l}1.000 \\
1.000 \\
1.000\end{array}$ & $\begin{array}{l}1 . \\
1 . \\
1 .\end{array}$ & $\begin{array}{l}1.000 \\
1.000 \\
1.000\end{array}$ \\
\hline $\begin{array}{l}.90 \\
.90 \\
.90\end{array}$ & $\begin{array}{l}\text { MM } \\
\text { MUU } \\
\text { MUT }\end{array}$ & $\begin{array}{l}.048 \\
.000 \\
.051\end{array}$ & $\begin{array}{l}.35 \\
.05 \\
.35\end{array}$ & $\begin{array}{l}.0 \\
.1 \\
.8\end{array}$ & 6 & $\begin{array}{r}1.000 \\
.954 \\
1.000\end{array}$ & $\begin{array}{r}1.0 \\
.9 \\
1.0\end{array}$ & $\begin{array}{l}1.000 \\
1.000 \\
1.000\end{array}$ & $\begin{array}{l}1.000 \\
1.000 \\
1.000\end{array}$ & $\begin{array}{l}00 \\
000 \\
000\end{array}$ & $\begin{array}{l}1 . \\
1 .\end{array}$ \\
\hline
\end{tabular}


POWER FOR 18 PAIRS

\begin{tabular}{|c|c|c|c|c|c|c|c|c|c|c|c|c|}
\hline \multicolumn{2}{|c|}{ METHOD } & .0 & .25 & .50 & .75 & 1.00 & 1.25 & 1.50 & 1.75 & 2.00 & 2.25 & \\
\hline $\begin{array}{l}.00 \\
.00 \\
.00 \\
.00\end{array}$ & $\begin{array}{l}\text { MM } \\
\text { MU } \\
\text { MUT } \\
\text { UU }\end{array}$ & $\begin{array}{l}.051 \\
.051 \\
.050 \\
.051\end{array}$ & $\begin{array}{l}.110 \\
.115 \\
.111 \\
.115\end{array}$ & $\begin{array}{l}.294 \\
.310 \\
.303 \\
.310\end{array}$ & $\begin{array}{l}.567 \\
.593 \\
.584 \\
.593\end{array}$ & $\begin{array}{l}.809 \\
.834 \\
.826 \\
.834\end{array}$ & $\begin{array}{l}.943 \\
.955 \\
.952 \\
.955\end{array}$ & $\begin{array}{l}.988 \\
.992 \\
.991 \\
.992\end{array}$ & $\begin{array}{l}.999 \\
.999 \\
.999 \\
.999\end{array}$ & $\begin{array}{l}1.000 \\
1.000 \\
1.000 \\
1.000\end{array}$ & $\begin{array}{l}1.000 \\
1.000 \\
1.000 \\
1.000\end{array}$ & 4 \\
\hline $\begin{array}{l}.10 \\
.10 \\
.10\end{array}$ & $\begin{array}{l}\text { MM } \\
\text { MU } \\
\text { MUT }\end{array}$ & $\begin{array}{l}.050 \\
.049 \\
.048\end{array}$ & $\begin{array}{l}.110 \\
.112 \\
.109\end{array}$ & $\begin{array}{l}.295 \\
.306 \\
.299\end{array}$ & $\begin{array}{l}.565 \\
.588 \\
.579\end{array}$ & $\begin{array}{l}.809 \\
.831 \\
.823\end{array}$ & $\begin{array}{l}.943 \\
.955 \\
.952\end{array}$ & $\begin{array}{l}.989 \\
.992 \\
.991\end{array}$ & $\begin{array}{r}.999 \\
.999 \\
.999\end{array}$ & $\begin{array}{l}1.000 \\
1.000 \\
1.000\end{array}$ & $\begin{array}{l}1.000 \\
1.000 \\
1.000\end{array}$ & \\
\hline $\begin{array}{l}.20 \\
.20 \\
.20\end{array}$ & $\begin{array}{l}\text { MM } \\
\text { MU } \\
\text { MUT }\end{array}$ & $\begin{array}{l}.050 \\
.046 \\
.046\end{array}$ & $\begin{array}{l}.112 \\
.109 \\
.108\end{array}$ & $\begin{array}{l}.306 \\
.307 \\
.303\end{array}$ & $\begin{array}{l}.583 \\
.595 \\
.588\end{array}$ & $\begin{array}{l}.826 \\
.838 \\
.832\end{array}$ & $\begin{array}{r}.951 \\
.958 \\
.956\end{array}$ & $\begin{array}{r}.991 \\
.993 \\
.992\end{array}$ & $\begin{array}{l}.999 \\
.999 \\
.999\end{array}$ & $\begin{array}{l}1.000 \\
1.000 \\
1.000\end{array}$ & $\begin{array}{l}1.000 \\
1.000 \\
1.000\end{array}$ & \\
\hline $\begin{array}{l}.30 \\
.30 \\
.30\end{array}$ & $\begin{array}{l}\text { MM } \\
\text { MU } \\
\text { MUT }\end{array}$ & $\begin{array}{l}.050 \\
.041 \\
.043\end{array}$ & $\begin{array}{l}.115 \\
.101 \\
.103\end{array}$ & $\begin{array}{l}.317 \\
.301 \\
.302\end{array}$ & $\begin{array}{l}.603 \\
.595 \\
.591\end{array}$ & $\begin{array}{l}.842 \\
.841 \\
.838\end{array}$ & $\begin{array}{l}.959 \\
.961 \\
.960\end{array}$ & $\begin{array}{r}.993 \\
.994 \\
.994\end{array}$ & $\begin{array}{r}.999 \\
.999 \\
.999\end{array}$ & $\begin{array}{l}1.000 \\
1.000 \\
1.000\end{array}$ & $\begin{array}{l}1.000 \\
1.000 \\
1.000\end{array}$ & \\
\hline $\begin{array}{l}.40 \\
.40 \\
.40\end{array}$ & $\begin{array}{l}\text { MM } \\
\text { MU } \\
\text { MUT }\end{array}$ & $\begin{array}{l}.051 \\
.034 \\
.040\end{array}$ & $\begin{array}{l}.120 \\
.093 \\
.103\end{array}$ & $\begin{array}{l}.337 \\
.293 \\
.305\end{array}$ & $\begin{array}{l}.638 \\
.597 \\
.605\end{array}$ & $\begin{array}{l}.871 \\
.852 \\
.854\end{array}$ & $\begin{array}{l}.971 \\
.966 \\
.966\end{array}$ & $\begin{array}{l}.996 \\
.995 \\
.995\end{array}$ & $\begin{array}{l}1.000 \\
1.000 \\
1.000\end{array}$ & $\begin{array}{l}1.000 \\
1.000 \\
1.000\end{array}$ & $\begin{array}{l}1.000 \\
1.000 \\
1.000\end{array}$ & \\
\hline $\begin{array}{l}.50 \\
.50 \\
.50\end{array}$ & $\begin{array}{l}\text { MM } \\
\text { MU } \\
\text { MUT }\end{array}$ & $\begin{array}{l}.050 \\
.026 \\
.039\end{array}$ & $\begin{array}{l}.128 \\
.081 \\
.105\end{array}$ & $\begin{array}{l}.372 \\
.284 \\
.323\end{array}$ & $\begin{array}{l}.687 \\
.603 \\
.635\end{array}$ & $\begin{array}{l}.902 \\
.863 \\
.877\end{array}$ & $\begin{array}{l}.982 \\
.973 \\
.975\end{array}$ & $\begin{array}{l}.998 \\
.997 \\
.997\end{array}$ & $\begin{array}{l}1.000 \\
1.000 \\
1.000\end{array}$ & $\begin{array}{l}1.000 \\
1.000 \\
1.000\end{array}$ & $\begin{array}{l}1.000 \\
1.000 \\
1.000\end{array}$ & $=$ \\
\hline $\begin{array}{l}.60 \\
.60 \\
.60\end{array}$ & $\begin{array}{l}\text { MM } \\
\text { MU } \\
\text { MUT }\end{array}$ & $\begin{array}{l}.050 \\
.017 \\
.041\end{array}$ & $\begin{array}{l}.143 \\
.065 \\
.118\end{array}$ & $\begin{array}{l}.422 \\
.268 \\
.363\end{array}$ & $\begin{array}{l}.756 \\
.609 \\
.692\end{array}$ & $\begin{array}{l}.942 \\
.881 \\
.914\end{array}$ & $\begin{array}{l}.993 \\
.981 \\
.987\end{array}$ & $\begin{array}{r}1.000 \\
.999 \\
.999\end{array}$ & $\begin{array}{l}1.000 \\
1.000 \\
1.000\end{array}$ & $\begin{array}{l}1.000 \\
1.000 \\
1.000\end{array}$ & $\begin{array}{l}1.000 \\
1.000 \\
1.000\end{array}$ & - \\
\hline $\begin{array}{l}.70 \\
.70 \\
.70\end{array}$ & $\begin{array}{l}\text { MM } \\
\text { MU } \\
\text { MUT }\end{array}$ & $\begin{array}{l}.050 \\
.008 \\
.046\end{array}$ & $\begin{array}{l}.169 \\
.048 \\
.152\end{array}$ & $\begin{array}{l}.510 \\
.252 \\
.463\end{array}$ & $\begin{array}{l}.845 \\
.624 \\
.798\end{array}$ & $\begin{array}{l}.977 \\
.903 \\
.961\end{array}$ & $\begin{array}{l}.999 \\
.989 \\
.996\end{array}$ & $\begin{array}{r}1.000 \\
.999 \\
1.000\end{array}$ & $\begin{array}{l}1.000 \\
1.000 \\
1.000\end{array}$ & $\begin{array}{l}1.000 \\
1.000 \\
1.000\end{array}$ & $\begin{array}{l}1.000 \\
1.000 \\
1.000\end{array}$ & \\
\hline $\begin{array}{l}.80 \\
.80 \\
.80\end{array}$ & $\begin{array}{l}\text { MM } \\
\text { MU.' } \\
\text { MUT }\end{array}$ & $\begin{array}{l}.050 \\
.002 \\
.052\end{array}$ & $\begin{array}{l}.218 \\
.029 \\
.217\end{array}$ & $\begin{array}{l}.656 \\
.223 \\
.642\end{array}$ & $\begin{array}{l}.941 \\
.644 \\
.928\end{array}$ & $\begin{array}{r}.997 \\
.930 \\
.994\end{array}$ & $\begin{array}{r}1.000 \\
.995 \\
1.000\end{array}$ & $\begin{array}{l}1.000 \\
1.000 \\
1.000\end{array}$ & $\begin{array}{l}1.000 \\
1.000 \\
1.000\end{array}$ & $\begin{array}{l}1.000 \\
1.000 \\
1.000\end{array}$ & $\begin{array}{l}1.000 \\
1.000 \\
1.000\end{array}$ & \\
\hline $\begin{array}{r}.90 \\
.90 \\
.90\end{array}$ & $\begin{array}{l}\text { MM } \\
\text { MU } \\
\text { MUT }\end{array}$ & $\begin{array}{l}.050 \\
.000 \\
.053\end{array}$ & $\begin{array}{l}.370 \\
.009 \\
.382\end{array}$ & $\begin{array}{l}.901 \\
.173 \\
.906\end{array}$ & $\begin{array}{l}.998 \\
.682 \\
.998\end{array}$ & $\begin{array}{r}1.000 \\
.968 \\
1.000\end{array}$ & $\begin{array}{r}1.000 \\
.999 \\
1.000\end{array}$ & $\begin{array}{l}1.000 \\
1.000 \\
1.000\end{array}$ & $\begin{array}{l}1.000 \\
1.000 \\
1.000\end{array}$ & $\begin{array}{l}1.000 \\
1.000 \\
1.000\end{array}$ & $\begin{array}{l}1.000 \\
1.000 \\
1.000\end{array}$ & \\
\hline
\end{tabular}


POWER FOR 19 PAIRS

\begin{tabular}{|c|c|c|c|c|c|c|c|c|c|c|c|}
\hline$\rho_{x y} \quad M$ & IOD & .0 & .25 & 50 & .75 & 1.00 & 1.25 & 1.50 & 1.75 & 2.00 & .25 \\
\hline $\begin{array}{l}.00 \\
.00 \\
.00 \\
.00\end{array}$ & $\begin{array}{l}\text { MM } \\
\text { MU } \\
\text { MUT } \\
\text { UU }\end{array}$ & $\begin{array}{l}.049 \\
.049 \\
.050 \\
.049\end{array}$ & $\begin{array}{l}.112 \\
.116 \\
.117 \\
.116\end{array}$ & $\begin{array}{l}.311 \\
.323 \\
.323 \\
.323\end{array}$ & $\begin{array}{l}.590 \\
.614 \\
.612 \\
.614\end{array}$ & $\begin{array}{l}.833 \\
.851 \\
.849 \\
.851\end{array}$ & $\begin{array}{r}.954 \\
.963 \\
.962 \\
.963\end{array}$ & $\begin{array}{r}.992 \\
.995 \\
.995 \\
.995\end{array}$ & $\begin{array}{r}.999 \\
.999 \\
.999 \\
.999\end{array}$ & $\begin{array}{l}1.000 \\
1.000 \\
1.000 \\
1.000\end{array}$ & $\begin{array}{l}1.000 \\
1.000 \\
1.000 \\
1.000\end{array}$ \\
\hline $\begin{array}{l}.10 \\
.10 \\
.10\end{array}$ & $\begin{array}{l}\text { MM } \\
\text { MU } \\
\text { MUT }\end{array}$ & $\begin{array}{l}.050 \\
.049 \\
.050\end{array}$ & $\begin{array}{l}.113 \\
.115 \\
.116\end{array}$ & $\begin{array}{l}.311 \\
.321\end{array}$ & $\begin{array}{l}.595 \\
.614 \\
.613\end{array}$ & $\begin{array}{l}.836 \\
.853 \\
.851\end{array}$ & $\begin{array}{l}.955 \\
.963 \\
.963\end{array}$ & $\begin{array}{l}.992 \\
.995 \\
.994\end{array}$ & $\begin{array}{r}.999 \\
.999 \\
.999\end{array}$ & & $\begin{array}{l}1.000 \\
1.000 \\
1.000\end{array}$ \\
\hline $\begin{array}{l}.20 \\
.20 \\
.20\end{array}$ & $\begin{array}{l}\text { MM } \\
\text { MU } \\
\text { MUT }\end{array}$ & $\begin{array}{l}.051 \\
.046 \\
.048\end{array}$ & $\begin{array}{l}.116 \\
.111 \\
.114\end{array}$ & $\begin{array}{l}.319 \\
.319 \\
.322\end{array}$ & $\begin{array}{l}.607 \\
.616 \\
.617\end{array}$ & $\begin{array}{l}.846 \\
.855 \\
.854\end{array}$ & $\begin{array}{l}.960 \\
.965 \\
.965\end{array}$ & $\begin{array}{r}.994 \\
.995 \\
.995\end{array}$ & $\begin{array}{r}.999 \\
.999 \\
.999\end{array}$ & & $\begin{array}{l}1.000 \\
1.000 \\
1.000\end{array}$ \\
\hline $\begin{array}{l}.30 \\
.30 \\
.30\end{array}$ & $\begin{array}{l}\text { MM } \\
\text { MU } \\
\text { MUT }\end{array}$ & $\begin{array}{l}.050 \\
.040 \\
.045\end{array}$ & $\begin{array}{l}.120 \\
.106 \\
.112\end{array}$ & $\begin{array}{l}.336 \\
.317 \\
.325\end{array}$ & $\begin{array}{l}.631 \\
.619 \\
.625\end{array}$ & $\begin{array}{l}.863 \\
.859 \\
.861\end{array}$ & $\begin{array}{l}.968 \\
.970 \\
.970\end{array}$ & $\begin{array}{l}.996 \\
.996 \\
.996\end{array}$ & & $\begin{array}{l}1.000 \\
1.000 \\
1.000\end{array}$ & $\begin{array}{l}1.000 \\
1.000 \\
1.000\end{array}$ \\
\hline $\begin{array}{l}.40 \\
.40 \\
.40\end{array}$ & $\begin{array}{l}\text { MM } \\
\text { MU } \\
\text { MUT }\end{array}$ & $\begin{array}{l}.050 \\
.033 \\
.042\end{array}$ & $\begin{array}{l}.125 \\
.095 \\
.108\end{array}$ & $\begin{array}{l}.355 \\
.308 \\
.327\end{array}$ & $\begin{array}{l}.665 \\
.623\end{array}$ & $\begin{array}{l}.888 \\
.870 \\
.877\end{array}$ & $\begin{array}{l}.977 \\
.973 \\
.974\end{array}$ & $\begin{array}{r}.997 \\
.997 \\
.997\end{array}$ & & & $\begin{array}{l}1.000 \\
1.000 \\
1.000\end{array}$ \\
\hline $\begin{array}{l}.50 \\
.50 \\
.50\end{array}$ & $\begin{array}{l}\text { MM } \\
\text { MU } \\
\text { MUT }\end{array}$ & $\begin{array}{l}.049 \\
.025 \\
.040\end{array}$ & $\begin{array}{l}.13 \\
.08 \\
.11\end{array}$ & $\begin{array}{l}.391 \\
.298 \\
.345\end{array}$ & $\begin{array}{l}.7 \\
.6\end{array}$ & $\begin{array}{l}.919 \\
.882 \\
.898\end{array}$ & $\begin{array}{r}.987 \\
.979 \\
.982\end{array}$ & $\begin{array}{l}.999 \\
.998 \\
.998\end{array}$ & & & $\begin{array}{l}1.000 \\
1.000 \\
1.000\end{array}$ \\
\hline $\begin{array}{l}.60 \\
.60 \\
.60\end{array}$ & $\begin{array}{l}\text { MM } \\
\text { MU } \\
\text { MUT }\end{array}$ & $\begin{array}{l}.049 \\
.016 \\
.042\end{array}$ & $\begin{array}{l}.149 \\
.069 \\
.126\end{array}$ & $\begin{array}{l}.45 \\
.28 \\
.39\end{array}$ & $\begin{array}{l}.038 \\
.726\end{array}$ & $\begin{array}{l}.954 \\
.898 \\
.931\end{array}$ & $\begin{array}{l}.995 \\
.986 \\
.991\end{array}$ & $\begin{array}{r}1.000 \\
.999 \\
.999\end{array}$ & & & $\begin{array}{l}1.000 \\
1.000 \\
1.000\end{array}$ \\
\hline $\begin{array}{l}.70 \\
.70 \\
.70\end{array}$ & $\begin{array}{l}\text { MM } \\
\text { MU } \\
\text { MUT }\end{array}$ & $\begin{array}{l}.050 \\
.007 \\
.048\end{array}$ & $\begin{array}{l}.175 \\
.051 \\
.162\end{array}$ & $\begin{array}{l}.530 \\
.269 \\
.491\end{array}$ & 8 & $\begin{array}{l}.983 \\
.920 \\
.971\end{array}$ & $\begin{array}{r}.999 \\
.992 \\
.998\end{array}$ & $\begin{array}{l}1.000 \\
1.000 \\
1.000\end{array}$ & $\begin{array}{l}1.000 \\
1.000 \\
1.000\end{array}$ & & $\begin{array}{l}1.000 \\
1.000 \\
1.000\end{array}$ \\
\hline $\begin{array}{l}.80 \\
.80\end{array}$ & $\begin{array}{l}\text { MM } \\
\text { MU } \\
\text { MUT }\end{array}$ & $\begin{array}{l}.049 \\
.002 \\
.053\end{array}$ & $\begin{array}{l}.229 \\
.030 \\
.234\end{array}$ & $\begin{array}{l}.679 \\
.239 \\
.672\end{array}$ & $\cdot 9$ & $\begin{array}{l}.998 \\
.947 \\
.997\end{array}$ & $\begin{array}{r}1.000 \\
.997 \\
1.000\end{array}$ & $\begin{array}{l}1.000 \\
1.000 \\
1.000\end{array}$ & $\begin{array}{l}1.000 \\
1.000 \\
1.000\end{array}$ & $\begin{array}{l}1.000 \\
1.000 \\
1.000\end{array}$ & $\begin{array}{l}1.000 \\
1.000 \\
1.000\end{array}$ \\
\hline $\begin{array}{l}.90 \\
.90\end{array}$ & $\begin{array}{l}\text { MM } \\
\text { MU } \\
\text { MUT }\end{array}$ & $\begin{array}{l}.050 \\
.000 \\
.055\end{array}$ & $\begin{array}{l}.387 \\
.009 \\
.405\end{array}$ & $\begin{array}{l}.916 \\
.191 \\
.923\end{array}$ & $\begin{array}{l}.999 \\
.721 \\
.999\end{array}$ & $\begin{array}{r}1.000 \\
.977 \\
1.000\end{array}$ & 1.000 & $\begin{array}{l}1.000 \\
1.000\end{array}$ & $\begin{array}{l}1.000 \\
1.000\end{array}$ & $\begin{array}{l}1.000 \\
1.000\end{array}$ & $\begin{array}{l}1.000 \\
1.000\end{array}$ \\
\hline
\end{tabular}

